$$
\begin{aligned}
\text { DO-TH } & 94-01 \\
& \sec 9405
\end{aligned}
$$

DO-TH $94 / 01$

January 1994

\title{
Polarized and unpolarized isolated prompt photon production beyond the leading order
}

\author{
L.E. Gordon and W. Vogelsang \\ Institut für Physik, Universität Dortmund \\ D-44221 Dortmund, Germany
}

\begin{abstract}
$\underline{\text { Abstract }}$
We present a simple and accurate analytical method for calculating the cross section for polarized and unpolarized isolated prompt photon production in next-to-leading order, taking into account also the next-to-leading order fragmentation contribution in the unpolarized case. We demonstrate the good accuracy of our method over a wide range of the isolation parameters and study the effects the isolation cuts have on the cross section at colliders.
\end{abstract}




\section{Introduction}

It has been recognized for a long time now that prompt photon production in hadronic collisions provides important tests of perturbative QCD and is very useful for constraining some of its most important parameters. For example the utility of this process for constraining the gluon distribution of the proton is well documented and in this context has been studied quite extensively [1-7]. More recently, interest in the possibility of using prompt photon production for longitudinal polarization of the proton beams in order to measure the polarized gluon distribution $\Delta G$ of the proton has been stimulated by the surprising result of the EMC measurement of the spin dependent proton structure function $g_{1}^{p}[8]$ and the more recent SMC and E142 Collaboration results on the neutron's $g_{1}^{n}[9,10]$. In this context, first leading order $\left(\mathcal{O}\left(\alpha \alpha_{s}\right)\right)$ studies [11] and more recently next-to-leading order $\left(\mathcal{O}\left(\alpha \alpha_{s}^{2}\right)\right)$ studies $[12,13,14]$ have been performed, examining the usefulness of (inclusive) polarized prompt photon production for settling the $\Delta G$ question [15]. The results of these studies have been mainly positive although in the case of the next-to-leading order calculations, lack of spin-dependent structure functions evolved in next-to-leading order has been a handicap.

In practice, when measuring the direct photon cross section at colliders, in contrast to the case of fixed target experiments, experimentalists must perform cuts in order to isolate the photon signal from the hadronic background. This is generally accomplished by requiring that the hadronic energy in a cone around the photon be less than a certain fraction of the photon energy. It is necessary in order to compare theory with experiment that the theoretical calculation incorporates the isolation criterion as much as possible.

In the following we outline an approximate method for calculating the isolated prompt photon cross section in next-to-leading order which is very quick and surprisingly accurate over quite a wide range of the isolation parameters. We develop this method for the 'direct' contributions from $a b \rightarrow \gamma c d, a, b, c$ and $d$ referring to partons, as well as for the next-to-leading order fragmentation contribution. We present our results, which are analytical formulas, for both the unpolarized and the longitudinally polarized case. Our calculation of the isolation effects for the unpolarized 'direct' contribution is partly based on the theoretical study of ref. [6] where a Monte Carlo program of [5] has been used to deal with the effects of isolation. We shall see that our analytical approximation method has some advantages over the Monte Carlo method. Our results for the next-to-leading 
order fragmentation piece and the polarized isolated cross section are entirely new. The rest of this paper is organized as follows. In section 2 we describe our method for dealing with isolation. More specifically, in subsection 2.1 we describe our method for calculating the effects of isolation cuts on the 'direct' (non-fragmentation) contributions. In section 2.2 we apply our method to the case of the fragmentation contribution in next-to-leading order. Section 2.3 shows the modifications to be made in order to obtain the corresponding results for the polarized case. In section 3 we present numerical results. We examine the accuracy of our results by comparing with Monte Carlo results and study the effects of isolation on the unpolarized prompt photon cross section. The appendices A-C contain our analytical results.

\section{Isolated Prompt Photon Production}

Experimentally, a prompt photon is considered as isolated if inside a cone of half-opening angle $\delta$ around the photon the sum of the energies of accompanying hadrons is less than $\epsilon E_{\gamma}$ where $E_{\gamma}$ is the photon's energy and $\epsilon$ is a small parameter of order $0.1[16,17]$. The parameter $\epsilon$ is generally called the energy resolution parameter and the cone around the photon is the isolation cone. Strictly speaking, the cone opening is defined by a radius $R$ of a circle, centered on the photon, in cms rapidity $(\eta)$ and azimuthal angle $(\phi)$ space via $R=\sqrt{(\Delta \eta)^{2}+(\Delta \phi)^{2}}$. For small rapidities of the prompt photon we have $R \approx \delta$.

In a leading order $\left(\mathcal{O}\left(\alpha \alpha_{s}\right)\right)$ theoretical study this experimental criterion can be easily implemented into the calculation. Here the dominant contributions arise from the hard $2 \rightarrow 2$ subprocesses $q g \rightarrow \gamma q$ and $q \bar{q} \rightarrow \gamma g$ for which the photon and the other final state particle, which will give rise to hadrons, are more or less back-to-back and thus separated from each other. Another leading order contribution comes from the fragmentation of a final state parton into a photon and is for a collision of two hadrons $A, B$ with momenta $p_{A}, p_{B}$ given by

$E_{\gamma} \frac{d^{3} \sigma_{f r a g}^{L O}}{d^{3} p_{\gamma}}=\frac{1}{\pi p_{T}^{2} S} \sum_{a b c d} \int_{z_{\min }}^{1} d z \int_{V W / z}^{1-(1-V) / z} d v f_{a}^{A}\left(x_{1}, M^{2}\right) f_{b}^{B}\left(x_{2}, M^{2}\right) \frac{\hat{s} d \hat{\sigma}^{a b \rightarrow c d}(\hat{s}, v)}{d v} D_{c}^{\gamma}\left(z, M_{f}^{2}\right)$

the sum correctly running over all $2 \rightarrow 2$ QCD subprocesses $a b \rightarrow c d$ with cross sections $d \hat{\sigma}^{a b \rightarrow c d}(\hat{s}, v) / d v$, and $D_{c}^{\gamma}\left(z, M_{f}^{2}\right)$ being the distribution function at scale $M_{f}$ for the fragmentation of parton-type $c$ into a photon which takes the momentum fraction $z$ of the 
parent parton. The functions $D_{c}^{\gamma}\left(z, M_{f}^{2}\right)$ are of order $\mathcal{O}\left(\alpha / \alpha_{s}\right)$ and thus make eq. (1) a leading order $\left(\mathcal{O}\left(\alpha \alpha_{s}\right)\right)$ contribution. In eq. (1), $f_{a}^{A}\left(x, M^{2}\right)$ denotes as usual the Bjørken$x$ distribution of parton $a$ in a hadron $A$ [18] at the scale $M^{2}$. Furthermore, introducing the transverse momentum $p_{T}$ of the prompt photon relative to the beam axis and the variable $x_{T} \equiv 2 p_{T} / \sqrt{S}$, where $S=\left(p_{A}+p_{B}\right)^{2}$, we have defined

$$
\begin{aligned}
1-V & \equiv \frac{x_{T}}{2} e^{-\eta}, \\
V W & \equiv \frac{x_{T}}{2} e^{\eta}, \\
z_{\min } & \equiv 1-V+V W, \\
x_{1} & \equiv \frac{V W}{z v}, \\
x_{2} & \equiv \frac{1-V}{z(1-v)}, \\
\hat{s} & \equiv x_{1} x_{2} S .
\end{aligned}
$$

Finally, the variable $v$ is connected with the $2 \rightarrow 2$ subprocess Mandelstam variable $\hat{t}_{1} \equiv\left(p_{a}-p_{c}\right)^{2}$ via

$$
\hat{t}_{1}=-\hat{s}(1-v)
$$

The effect of isolation on eq. (1) is simply the additional constraint [6]

$$
z \geq \frac{1}{1+\epsilon}
$$

which converts $z_{\min }$ into

$$
z_{\min }=\max \left(1-V+V W, \frac{1}{1+\epsilon}\right),
$$

and the choice of a $\delta$-dependent scale $[6]$

$$
M_{f} \sim \mathcal{O}\left(\delta p_{T}\right)
$$

instead of $M_{f} \sim \mathcal{O}\left(p_{T}\right)$ for the non-isolated case. The constraint (4) usually leads to a strong reduction of the size of the fragmentation contribution.

In next-to-leading order $\left(\mathcal{O}\left(\alpha \alpha_{s}^{2}\right)\right)$ important contributions to prompt photon production arise from the various possible 'direct' $2 \rightarrow 3$ processes $a b \rightarrow \gamma c d$. In contrast to the leading order processes $q g \rightarrow \gamma q$ and $q \bar{q} \rightarrow \gamma g$ it is now possible kinematically that one of the final state partons carrying more energy than $\epsilon E_{\gamma}$ happens to be inside the cone around the photon (fig. 1a). As mentioned above, such contributions have to be excluded for the isolated cross section. 
In a complete and consistent next-to-leading order calculation one also has to take into account the fragmentation contribution beyond the leading order which turns out to be rather important at small $x_{T}$. Here there are contributions from all $2 \rightarrow 3$ QCD processes where one of the outgoing partons fragments into a photon. For the isolated cross section the requirement that the remaining hadronic energy after fragmentation be restricted by $\epsilon E_{\gamma}$ again leads to the cut (4). This cut is, however, in general not sufficient if a non-fragmenting parton from the $2 \rightarrow 3 \mathrm{QCD}$ process is also radiated into the cone around the photon which is of course possible kinematically (fig. 1b). This parton gives rise to additional hadronic energy accompanying the photon which invalidates eq. (4).

In the following two subsections we will present a simple, yet very accurate, approximation method for dealing with the isolation of prompt photons in next-to-leading order. Subsection 2.1 deals with isolating the 'direct' contributions from $a b \rightarrow \gamma c d$, whereas subsection 2.2 is devoted to an analysis of the isolated fragmentation contribution. We note that there is a Monte Carlo program by Baer et al. [5] which is able to deal with the isolation of the direct contribution numerically and thus 'exactly'. Nevertheless we think that our approximation method has several advantages. Firstly, it is faster by orders of magnitude since it is based on simple analytic expressions which only need to be convoluted with parton distributions. Secondly, it does not suffer from the large numerical fluctuations inherent to the Monte Carlo calculation which were also observed in ref. [6]. Moreover, the study of the effects of isolation on the next-to-leading order fragmentation contribution, which have to be included for a consistent calculation, is entirely new. As for this contribution, a Monte Carlo calculation would certainly be too difficult and computer-time consuming, and our approximation method probably offers the only viable alternative.

In section 2.3 we shall present the corresponding results for longitudinal polarization of the incoming hadrons. These results will probably be important for future polarized high energy colliders such as RHIC [19].

\subsection{Isolation cuts on the 'direct' contributions $a b \rightarrow \gamma c d$}

Apart from the Monte Carlo program of Baer et al. [5] there exist two analytical calculations of the complete next-to-leading order corrections for the 'direct' (hard) part of inclusive prompt photon production, i.e. of the processes $a b \rightarrow \gamma c d[2,3,13]$. These 
calculations have been performed integrating over the full phase space of the outgoing unobserved particles $c$ and $d$, and thus no longer allow for isolation cuts directly. Nevertheless they present the most convenient starting point for the treatment of the isolated 'direct' cross section since the latter can be written as the inclusive cross section minus a subtraction piece [6]:

$$
E_{\gamma} \frac{d^{3} \sigma_{d i r}^{i s o l}}{d^{3} p_{\gamma}}=E_{\gamma} \frac{d^{3} \sigma_{d i r}^{\text {incl }}}{d^{3} p_{\gamma}}-E_{\gamma} \frac{d^{3} \sigma_{d i r}^{s u b}}{d^{3} p_{\gamma}}
$$

$E_{\gamma} d^{3} \sigma_{d i r}^{s u b} / d^{3} p_{\gamma}$ being the cross section for producing a prompt photon with energy $E_{\gamma}$ which is accompanied by hadronic energy more than $\epsilon E_{\gamma}$ inside the cone. The decomposition of $E_{\gamma} d^{3} \sigma_{d i r}^{i s o l} / d^{3} p_{\gamma}$ in eq. (7) has several advantages. First of all, the inclusive cross section $E_{\gamma} d^{3} \sigma_{d i r}^{i n c l} / d^{3} p_{\gamma}$ is perturbatively well defined in itself in the sense that a complete cancellation of all poles has already taken place, i.e. infrared poles have cancelled between the virtual $(2 \rightarrow 2)$ and the $2 \rightarrow 3$ next-to-leading order contributions and mass singularities have been factored into the initial state parton distributions and the photon fragmentation functions $[2,3,13]$. Furthermore, as we shall soon see, it is possible to give a simple approximation for the subtraction cross section $E_{\gamma} d^{3} \sigma_{d i r}^{s u b} / d^{3} p_{\gamma}$ which is nevertheless very accurate.

Before starting the calculation of $E_{\gamma} d^{3} \sigma_{d i r}^{s u b} / d^{3} p_{\gamma}$ let us note that in the calculation of this cross section one also encounters mass singularities coming from the collinear emission of the photon by an outgoing quark [20], just as this was the case in the calculation of the next-to-leading order inclusive cross section $E_{\gamma} d^{3} \sigma_{d i r}^{i n c l} / d^{3} p_{\gamma}$ before factorization of the final state singularities. In the latter case these singularities were absorbed into the photon fragmentation functions $D_{q}^{\gamma}\left(z, M_{f}^{2}\right)$, and the same step has to be taken for the final state singularities in $E_{\gamma} d^{3} \sigma_{d i r}^{s u b} / d^{3} p_{\gamma}$. To be more precise, let us first note that the collinear singularities in the difference $E_{\gamma} d^{3} \sigma_{d i r}^{i s o l} / d^{3} p_{\gamma}=E_{\gamma} d^{3} \sigma_{d i r}^{i n c l} / d^{3} p_{\gamma}-E_{\gamma} d^{3} \sigma_{d i r}^{s u b} / d^{3} p_{\gamma}$ must cancel each other for $\epsilon \rightarrow 0$ because in this limit there is no final state parton in the cone the photon could become collinear with. For $\epsilon \rightarrow 0$ the photon would become completely isolated [21]. Thus, working in dimensional regularization with $n=4-2 \tau$ space-time dimensions in order to extract the singularities, the pole structure of $E_{\gamma} d^{3} \sigma_{d i \tau}^{\text {incl }} / d^{3} p_{\gamma}-E_{\gamma} d^{3} \sigma_{d i \tau}^{s u b} / d^{3} p_{\gamma}$ is, before final state factorization, for small $\epsilon$ schematically given by

$$
\begin{aligned}
\left.E_{\gamma} \frac{d^{3} \sigma_{d i r}^{i s o l}}{d^{3} p_{\gamma}}\right|_{p o l e} & =\left.E_{\gamma} \frac{d^{3} \sigma_{d i r}^{i n c l}}{d^{3} p_{\gamma}}\right|_{p o l e}-\left.E_{\gamma} \frac{d^{3} \sigma_{d i r}^{s u b}}{d^{3} p_{\gamma}}\right|_{p o l e} \\
& \sim-\frac{\epsilon}{\tau} \mu^{2 \tau} \sum_{a b q d} \frac{d \hat{\sigma}^{a b \rightarrow q d}}{d v} \otimes f_{a}\left(x_{1}, M^{2}\right) f_{b}\left(x_{2}, M^{2}\right) \otimes \alpha P_{\gamma q}(z),
\end{aligned}
$$

where the ' $\otimes$ ' denote proper convolutions (integrations), the precise form of which is im- 
material for our present purpose, $P_{\gamma q}(z)=\left(1+(1-z)^{2}\right) / z$ is the quark-to-photon splitting function, and $\mu$ an arbitrary mass scale. It becomes obvious that the structure of eq. (8) corresponds to that of eq. (1) when the limit (4), $z \geq 1 /(1+\epsilon)$, is implemented for small $\epsilon$. The pole term (8) can thus be factored at some scale $M_{f} \sim \delta p_{T}[6]$ into the fragmentation contribution (1), giving rise to 'dressed' photon fragmentation functions $D_{q}^{\gamma}\left(z, M_{f}^{2}\right)$ which obey a next-to-leading order QCD evolution equation. In practice, we do not have to proceed via eq. (8) to factorize the poles appearing in the calculation of $E_{\gamma} d^{3} \sigma_{d i r}^{s u b} / d^{3} p_{\gamma}$. As mentioned above, we want to use our previous results [13] for inclusive prompt photon production $\left(E_{\gamma} d^{3} \sigma_{d i r}^{i n c l} / d^{3} p_{\gamma}\right)$ as the starting point for our calculation. The results for $E_{\gamma} d^{3} \sigma_{d i r}^{i n c l} / d^{3} p_{\gamma}$ in [13] are of course completely factorized, i.e. do not contain any poles. Therefore, in the calculation of $E_{\gamma} d^{3} \sigma_{d i r}^{s u b} / d^{3} p_{\gamma}$ we can simply subtract the pole terms in the same way they were factorized in the calculation of $E_{\gamma} d^{3} \sigma_{d i r}^{\text {incl }} / d^{3} p_{\gamma}$, keeping in mind that the pole terms in $E_{\gamma} d^{3} \sigma_{d i r}^{i n c l} / d^{3} p_{\gamma}$ and $E_{\gamma} d^{3} \sigma_{d i r}^{s u b} / d^{3} p_{\gamma}$ are in principle factorized along with each other via eq. (8). It is of course crucial to perform the subtraction of the poles in $E_{\gamma} d^{3} \sigma_{d i \tau}^{s u b} / d^{3} p_{\gamma}$ in the same manner as in $E_{\gamma} d^{3} \sigma_{d i r}^{i n c l} / d^{3} p_{\gamma}$. This means, in accordance to our previous calculation [13], that we have to work in dimensional regularization and to use the same factorization scheme, namely the $\overline{\mathrm{MS}}$-scheme [22], for the subtraction of the collinear singularities. As was pointed out in ref. [13], it is straightforward to transform our results (i.e. those for $E_{\gamma} d^{3} \sigma_{d i r}^{i n c l} / d^{3} p_{\gamma}$ and for $E_{\gamma} d^{3} \sigma_{d i r}^{s u b} / d^{3} p_{\gamma}$ ) to another factorization scheme.

We perform our actual calculation of $E_{\gamma} d^{3} \sigma_{d i r}^{s u b} / d^{3} p_{\gamma}$ in the hadronic cms since this is the relevant frame for collider experiments. In order to find a semi-analytical expression for $E_{\gamma} d^{3} \sigma_{d i r}^{s u b} / d^{3} p_{\gamma}$, we shall assume that $\delta$ is small, i.e. that the cone around the photon, needed for isolating it, is rather narrow [23]. As we shall see below, the leading behavior of $E_{\gamma} d^{3} \sigma_{d i r}^{s u b} / d^{3} p_{\gamma}$ for small $\delta$ is logarithmic in $\delta$ which is a remnant of the final state collinear singularities arising in the calculation of $E_{\gamma} d^{3} \sigma_{d i r}^{\text {sub }} / d^{3} p_{\gamma}$. We shall also consistently keep terms constant with respect to $\delta$, since these turn out to be of numerical relevance. According to our previous discussion they are furthermore needed since they contain the dependence on the factorization scheme which must be the same in the calculation of $E_{\gamma} d^{3} \sigma_{d i r}^{s u b} / d^{3} p_{\gamma}$ and $E_{\gamma} d^{3} \sigma_{d i r}^{i n c l} / d^{3} p_{\gamma}$. All remaining pieces in the subtraction cross section are suppressed by powers of $\delta$ and are negligible. The only exception from this occurs when $\epsilon$ becomes very small. In this case the subtraction cross section is dominated by soft gluons being radiated into the cone which give rise to a logarithmic dependence on $\epsilon$ and eventually lead to an infrared divergence at $\epsilon=0$ [6]. The reason for this is simple: 
A completely isolated cross section, with no hadronic energy at all in the isolation cone, is not a perturbatively well-defined quantity for a massless particle [6]. Although in reality $\epsilon$ is fixed by the experimental resolution, it is necessary to keep the contributions which are logarithmically dependent on $\epsilon$ in order to improve the accuracy of the approximation for the subtraction piece. Thus schematically we have the following structure of our approximated subtraction cross section:

$$
E_{\gamma} \frac{d^{3} \sigma_{d i r}^{s u b}}{d^{3} p_{\gamma}}=\mathcal{A} \ln \delta+\mathcal{B}+\mathcal{C} \delta^{2} \ln \epsilon
$$

where the coefficients $\mathcal{A}, \mathcal{B}$ and $\mathcal{C}$ are functions of the kinematical variables. Note that $\mathcal{A}$ and $\mathcal{B}$ also depend on $\epsilon$ due to the isolation cuts. It is now our purpose to extract the coefficient functions $\mathcal{A}, \mathcal{B}$ and $\mathcal{C}$. The contributing subprocesses are the same as in the completely inclusive calculation $\left(E_{\gamma} d^{3} \sigma_{d i r}^{i n c l} / d^{3} p_{\gamma}\right)[13]$, namely $q \bar{q} \rightarrow \gamma g g, q g \rightarrow \gamma q g$, $g g \rightarrow \gamma q \bar{q}, q q \rightarrow \gamma q q, q \bar{q} \rightarrow \gamma q \bar{q}, q \bar{q} \rightarrow \gamma q^{\prime} \bar{q}^{\prime}$ and $q q^{\prime} \rightarrow \gamma q q^{\prime}$. Only the processes with (anti)quarks in the final state can lead to contributions to $\mathcal{A}$ and $\mathcal{B}$ whereas, as discussed above, only the first two subprocesses which involve gluon radiation can give $\ln \epsilon$ terms and contribute to $\mathcal{C}$.

For small $\delta$ (small-cone approximation) the $n=4-2 \tau$-dimensional phase space for the process $a\left(p_{1}\right) b\left(p_{2}\right) \rightarrow \gamma\left(k_{1}\right) c\left(k_{2}\right) d\left(k_{3}\right)$ with parton $c$ being inside a cone of half-angle $\delta$ around the photon reads in the hadronic cms frame [25]

$$
\begin{aligned}
\frac{d R_{3}}{d v d w} & =\frac{\hat{s}}{(4 \pi)^{4} \Gamma(1-2 \tau)}\left(\frac{4 \pi}{\hat{s}}\right)^{2 \tau}\left(\frac{1-V+V W}{1-v+v w}\right)^{2(1-\tau)}\left(\frac{v w(1-v)}{V W(1-V)}\right)^{1-\tau} \\
& \times v^{2-3 \tau}(w(1-v))^{-\tau}(1-w)^{1-2 \tau} \int_{0}^{\pi} d \theta_{2} \sin ^{-2 \tau} \theta_{2} \int_{0}^{\delta} d \theta_{1} \theta_{1}^{1-2 \tau}
\end{aligned}
$$

where we have defined

$$
\begin{aligned}
v & \equiv 1+\frac{\hat{t}_{1}}{\hat{s}}, \\
w & \equiv-\frac{\hat{u}_{1}}{\hat{s}+\hat{t}_{1}}
\end{aligned}
$$

with $\hat{t}_{1}=\left(p_{1}-k_{1}\right)^{2}, \hat{u}_{1}=\left(p_{2}-k_{1}\right)^{2}$, where $p_{1}, p_{2}$ and $k_{1}$ are the momenta of the incoming partons and the photon. Furthermore, $\theta_{1}$ and $\theta_{2}$ are the polar and azimuthal angles of $k_{2}$ with respect to $k_{1}$, i.e.

$$
\begin{aligned}
& k_{1}=E_{\gamma}(1,1,0, \ldots, 0) \\
& k_{2}=E_{2}\left(1, \cos \theta_{1}, \sin \theta_{1} \cos \theta_{2}, \ldots\right)
\end{aligned}
$$


In the small-cone approximation, $\delta \rightarrow 0$, we can usually set $\theta_{1} \approx 0$, i.e. $\sin \theta_{1} \approx 0$ and $\cos \theta_{1} \approx 1$, in the matrix elements for $a b \rightarrow \gamma c d$. Then we have

$$
k_{2} \approx \rho k_{1}
$$

with

$$
\rho=E_{2} / E_{\gamma}=\frac{v(1-w)}{1-v+v w} .
$$

Imposing the collinear kinematics (11), (12) on the matrix elements simplifies these significantly. Only when the inverse of

$$
s_{12}=\left(k_{1}+k_{2}\right)^{2}=2 E_{\gamma} E_{2}\left(1-\cos \theta_{1}\right)
$$

appears do we have to keep a finite $\theta_{1}$. Terms $\sim 1 / s_{12}$ (which only appear if particle 2 is a quark or antiquark) lead to an angular integral of the type [25]

$$
\int_{0}^{\pi} d \theta_{2} \sin ^{-2 \tau} \theta_{2} \int_{0}^{\delta} d \theta_{1} \frac{\theta_{1}^{1-2 \tau}}{1-\cos \theta_{1}} \approx-\frac{1}{\tau} B\left(\frac{1}{2}-\tau, \frac{1}{2}\right) \delta^{-2 \tau}\left(1+\mathcal{O}\left(\delta^{2}\right)\right)
$$

(where $B(x, y)$ is the Beta function), which gives rise to the expected collinear singularities. As discussed above, these are removed by the factorization procedure. The factor $\delta^{-2 \tau}$ in eq. (13), when expanded in powers of $\tau$, leads to a logarithmic behavior of the subtraction cross section for small $\delta$, i.e. allows for the extraction of the coefficient function $\mathcal{A}$ in eq. (9). It is also straightforward to keep all terms having a constant behavior with respect to $\delta$, which are the terms corresponding to $\mathcal{B}$ in eq. (9). These can arise only from terms $\sim \tau / s_{12}$ in the matrix elements, from factors like $(1-w)^{1-2 \tau}$ in the expression for the $n$-dimensional $2 \rightarrow 3$ phase space (10), or from finite (with respect to $\tau \rightarrow 0$ ) contributions from factorization. As can be easily seen, all other terms in the matrix elements are suppressed by powers of $\delta$ after phase space integration.

The general structure of the final result for $E_{\gamma} d^{3} \sigma_{d i r}^{s u b} / d^{3} p_{\gamma}$ (disregarding for the moment the $\delta^{2} \ln \epsilon$ pieces) can be anticipated. After imposing the collinear limit on the various $2 \rightarrow 3$ matrix elements containing quarks in the final state and integrating over phase space via eq. (13) one obtains

$$
\int|M|^{2} \frac{d R_{3}}{d v d w} \sim-\frac{(\mu \delta)^{2 \tau}}{\tau} \tilde{P}_{\gamma q}(1-v+v w, \tau) \frac{d \tilde{\hat{\sigma}}^{a b \rightarrow q d}}{d v}\left(\hat{s}, \frac{v w}{1-v+v w}, \tau\right)
$$

where

$$
\tilde{P}_{\gamma q}(z, \tau)=\frac{1+(1-z)^{2}}{z}-\tau z
$$


is the $n$-dimensional splitting function for the quark-to-photon transition, and the $d \tilde{\hat{\sigma}}^{a b \rightarrow q d}(\hat{s}, y, \tau) / d y$ are the cross sections for the processes $a b \rightarrow q d$ in $n$ dimensions. $\overline{\mathrm{MS}}$ factorization is carried out by subtracting a term proportional to

$$
\sim-\frac{1}{\tau}\left(\frac{\mu^{2}}{M_{f}^{2}}\right)^{\tau} P_{\gamma q}(1-v+v w) \frac{d \tilde{\hat{\sigma}}^{a b \rightarrow q d}}{d v}\left(\hat{s}, \frac{v w}{1-v+v w}, \tau\right),
$$

where $P_{\gamma q}(z)$ is the four-dimensional splitting function to be obtained by setting $\tau=0$ in eq. (15). Taking the difference of eqs. (14) and (16) and expanding in $\tau$ the pole terms cancel out as well as all terms proportional to $\tau$ in $d \tilde{\hat{\sigma}}^{a b \rightarrow q d}(\hat{s}, y, \tau) / d y$ which are equally present in eqs. (14) and (16). This is not true, however, for the $\tau$-dependent terms in the quark-to-photon splitting functions in eqs. (14) and (16). Thus the structure of the final factorized result for $E_{\gamma} d^{3} \sigma_{d i r}^{s u b} / d^{3} p_{\gamma}$ (without the $\delta^{2} \ln \epsilon$ pieces) is the following:

$$
E_{\gamma} \frac{d^{3} \sigma_{d i r}^{s u b}}{d^{3} p_{\gamma}} \sim \sum_{a b q d} \frac{d \hat{\sigma}^{a b \rightarrow q d}}{d v} \otimes f_{a}\left(x_{1}, M^{2}\right) f_{b}\left(x_{2}, M^{2}\right) \otimes \alpha \mathcal{P}_{\gamma q}(z)
$$

with

$$
\mathcal{P}_{\gamma q}(z)=\frac{1+(1-z)^{2}}{z} \ln \frac{v^{2}(1-w)^{2} E_{\gamma}^{2} \delta^{2}}{M_{f}^{2}}+z
$$

the logarithmic piece being a remainder of the $1 / \tau$ poles, and the nonlogarithmic piece corresponding to the term proportional to $\tau$ in eq. (15). Eq. (18) shows another reason why it is crucial to keep the terms constant with respect to $\delta$ : Choosing a scale of order $\delta p_{T}$ eliminates $\delta$ from the logarithm in eq. (18) turning this logarithm into a term which is also constant with respect to $\delta$. Explicitly our results read

$$
\left.E_{\gamma} \frac{d^{3} \sigma_{d i}^{s u b}}{d^{3} p_{\gamma}}\right|_{1}=\frac{1}{\pi p_{T}^{4}} \sum_{a b} \int_{V W+\frac{\epsilon}{1+\epsilon}}^{V} d v \int_{V W / v}^{1-\frac{\epsilon}{v(1+c)}} d w x_{1} f_{a}\left(x_{1}, M^{2}\right) x_{2} f_{b}\left(x_{2}, M^{2}\right) v w(1-v) \hat{s} \frac{d \hat{\sigma}_{\delta}^{a b}}{d v d w}
$$

where

$$
\begin{aligned}
& x_{1}=\frac{V W}{v w}, \\
& x_{2}=\frac{1-V}{1-v}
\end{aligned}
$$

and the subscript ' 1 ' indicates that eq. (19) does not contain the $\ln \epsilon$ pieces. The integration limits in eq. (19) stem from the condition which defines our subtraction cross section, namely

$$
E_{2}=E_{\gamma} \frac{v(1-w)}{1-v+v w} \geq \epsilon E_{\gamma}
$$


For the various subprocesses we have [27]

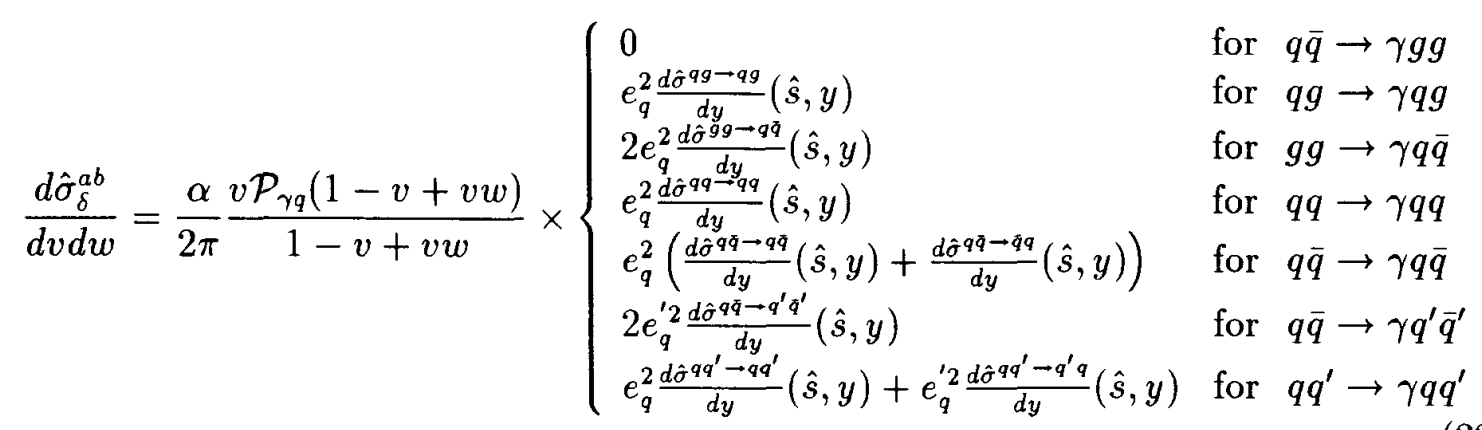

where $y=v w /(1-v+v w)$ and $e_{q}$ and $e_{q}^{\prime}$ are the charges of $q$ and $q^{\prime}$. The final states in eq. (22) have been properly symmetrized in order to account for the possibility of particle $d$ radiating the photon in the reaction $a b \rightarrow \gamma c d$. For convenience we list the cross sections $d \hat{\sigma}^{a b \rightarrow c d}(\hat{s}, y) / d y$ in appendix A.

Let us now turn to the extraction of the $\ln \epsilon$ pieces. This problem was already partly considered in ref. [6]. As mentioned before, the $\ln \epsilon$ pieces arise from soft gluons being radiated into the cone, i.e. they can only come from the $q \bar{q} \rightarrow \gamma g g$ and $q g \rightarrow \gamma q g$ subprocesses which have final state gluons. The procedure is as before: We consider the $2 \rightarrow 3$ matrix elements in the limit when a final state gluon becomes collinear to the outgoing prompt photon. In contrast to quarks being collinear to the photon, no $1 / \tau$-poles can arise from gluons inside the cone since there is no direct photon gluon coupling. This means that the $\ln \epsilon$ terms are not accompanied by $\ln \delta$ factors. It is therefore sufficient to consider the $2 \rightarrow 3$ matrix elements in 4 dimensions. Instead of eq. (13) we only need the trivial phase space integral

$$
\int_{\delta} d \Omega \equiv \int_{0}^{\pi} d \theta_{2} \int_{0}^{\delta} \theta_{1} d \theta_{1}=\frac{\delta^{2} \pi}{2}
$$

which shows that the $\ln \epsilon$ pieces are suppressed by $\delta^{2}$ [28] for small $\delta$. As was noted in [6] the logarithms of $\epsilon$ arise from terms $\sim 1 / E_{g}$ in the matrix elements, where $E_{g}$ is the gluon energy. Since according to eq. (12) $E_{g} \sim(1-w)$ in the collinear limit, we find that the matrix elements have the structure $f(v, w) /(1-w)$ in the small-cone approximation after phase space integration, where $f(v, w)$ is a function regular at $w=1$. The integration limits for the $v$ and the $w$ integration are as in eq. (19). For small $\epsilon$ we may write

$$
\int_{V W+\frac{\epsilon}{1+\varepsilon}}^{V} d v \int_{V W / v}^{1-\frac{\epsilon}{v(1+\epsilon)}} d w \frac{f(v, w)}{1-w} \approx-\int_{V W}^{V} d v f(v, 1) \ln \left(\frac{\epsilon}{v-V W}\right)
$$

where, apart from the leading $\ln \epsilon$ term, we have kept a non-leading term in the logarithm which derives from the lower limit of the $w$ integration and turns out to be crucial for 
a good accuracy if $\epsilon$ is not very small. The reason for this is that we are dealing with the subtraction cross section: In principle we want to subtract all contributions from gluons being inside the cone around the photon with energy larger than $\epsilon E_{\gamma}$ (see eq. (7)). This can be both soft $(w \rightarrow 1)$ and hard ( $w$ small) gluons, which explains that the presence of the lower $w$ integration limit in the logarithm in eq. (24) is needed for a good approximation. Nevertheless, we do not try to consistently keep the other non-leading contributions (with respect to $\epsilon$ ) since these can also arise from quarks being inside the cone, i.e. from terms in the matrix elements which do not behave like $1 /(1-w)$. We therefore anticipate that our approximation is probably better with respect to $\delta$ (where we were able to consistently keep all constant pieces) than it is with respect to $\epsilon$. However, it turns out in the numerical evaluation (i.e. by comparing with Monte Carlo results) that the $\ln \epsilon$ pieces are of minor importance for the subtraction cross section over a wide range of $\epsilon$ and $\delta$.

We now give our final result for the $\ln \epsilon$ contributions (i.e. the coefficient $\mathcal{C}$ in eq. (9)):

$$
\left.E_{\gamma} \frac{d^{3} \sigma_{d i r}^{s u b}}{d^{3} p_{\gamma}}\right|_{2}=\frac{1}{\pi p_{T}^{4}} \sum_{a b} \int_{V W}^{V} d v x_{1} f_{a}\left(x_{1}, M^{2}\right) x_{2} f_{b}\left(x_{2}, M^{2}\right) v(1-v) \hat{s} \frac{d \hat{\sigma}_{\epsilon}^{a b}}{d v}
$$

where

$$
v(1-v) \hat{s} \frac{d \hat{\sigma}_{\epsilon}^{a b}}{d v}=-\frac{E_{\gamma}^{2} \delta^{2}}{\hat{s}} \ln \left(\frac{\epsilon}{v-V W}\right) \times\left\{\begin{array}{cl}
\frac{2 C_{F}}{N_{C}} \frac{C_{F}-N_{C} v v_{1}}{v v_{1}} T_{q \bar{q}} & \text { for } q \bar{q} \rightarrow \gamma g g \\
\frac{1}{N_{C}} \frac{C_{F} v^{2}+N_{C} v_{1}}{v_{1}} T_{q g} & \text { for } q g \rightarrow \gamma q g \\
0 & \text { otherwise }
\end{array}\right.
$$

with $v_{1}=1-v$ and

$$
\begin{aligned}
& T_{q \bar{q}}=v^{2}+(1-v)^{2} \\
& T_{q g}=1+(1-v)^{2}
\end{aligned}
$$

which are essentially the Born cross sections for prompt photon production. Furthermore, $x_{1}$ and $x_{2}$ are defined as in eq. (2), setting $z=1$ there. The color structure of $d \hat{\sigma}_{\epsilon}^{a b} / d v$ for $q \bar{q}(q g)$ scattering stems from the fact that the gluon can be radiated collinear to the photon at various possible legs in the process $q \bar{q} \rightarrow \gamma g(g)(q g \rightarrow \gamma q(g))$. Adding up $E_{\gamma} d^{3} \sigma_{d i r}^{s u b} /\left.d^{3} p_{\gamma}\right|_{1}$ and $E_{\gamma} d^{3} \sigma_{d i r}^{s u b} /\left.d^{3} p_{\gamma}\right|_{2}$ from eqs. (19) and (25) gives the final expression for the subtraction cross section and completes our calculation of the isolation effects in the direct reactions $a b \rightarrow \gamma c d$. Inserting $E_{\gamma} d^{3} \sigma_{d i r}^{s u b} /\left.d^{3} p_{\gamma}\right|_{1}+E_{\gamma} d^{3} \sigma_{d i r}^{s u b} /\left.d^{3} p_{\gamma}\right|_{2}$ together with

$$
E_{\gamma} \frac{d^{3} \sigma_{d i \tau}^{\text {incl }}}{d^{3} p_{\gamma}}=\frac{1}{\pi p_{T}^{4}} \sum_{a b} \int_{V W}^{V} d v \int_{V W / v}^{1} d w x_{1} f_{a}\left(x_{1}, M^{2}\right) x_{2} f_{b}\left(x_{2}, M^{2}\right) v w(1-v) \hat{s} \frac{d \hat{\sigma}^{a b}}{d v d w}
$$


into eq. (7) yields the direct piece of the isolated prompt photon cross section. The subprocess cross sections $d \hat{\sigma}^{a b} / d v d w$ for the inclusive cross section were published in a compact analytical form in ref. [13].

\subsection{Isolation cuts on the next-to-leading order fragmentation contribution}

In this section we want to extend our method to the case of next-to-leading order fragmentation. The procedure is quite similar to that outlined in the last subsection. Again it is very convenient to start by introducing a subtraction piece. In eq. (4) we have introduced a cut on the fragmentation variable $z$ which expresses that the remaining hadronic energy after fragmentation is restricted by $\epsilon E_{\gamma}$. As we pointed out in the beginning of section 2 , the cut (4) is in general no longer sufficient if a non-fragmenting parton is also radiated into the cone (fig. $1 \mathrm{~b}$ ) since this gives rise to additional hadronic energy. If this happens we have to make sure that the sum of the energies of the fragmentation remnants plus the energy of the additional non-fragmenting particle be smaller than $\epsilon E_{\gamma}$. We therefore set up our calculation of the isolated next-to-leading order fragmentation contribution $E_{\gamma} d^{3} \sigma_{f r a g}^{i s o l} / d^{3} p_{\gamma}$ in the following way:

$$
E_{\gamma} \frac{d^{3} \sigma_{f r a g}^{i s o l}}{d^{3} p_{\gamma}}=E_{\gamma} \frac{d^{3} \sigma_{f r a g}^{z \geq 1 /(1+\epsilon)}}{d^{3} p_{\gamma}}-E_{\gamma} \frac{d^{3} \sigma_{f r a g}^{\text {sub }}}{d^{3} p_{\gamma}},
$$

where $E_{\gamma} d^{3} \sigma_{f r a g}^{z \geq 1 /(1+\epsilon)} / d^{3} p_{\gamma}$ is the next-to-leading order fragmentation cross section with the (insufficient) $z$-cut (4) implemented, and $E_{\gamma} d^{3} \sigma_{f r a g}^{s u b} / d^{3} p_{\gamma}$ is the fragmentation subtraction cross section which is the cross section for an additional particle being inside the cone, satisfying the conditions

$$
\begin{aligned}
E_{f r a g}^{r e m} & \leq \epsilon E_{\gamma} \quad \text { (equivalent to eq. (4)) } \\
\text { but } E_{f r a g}^{r e m}+E_{2} & \geq \epsilon E_{\gamma}
\end{aligned}
$$

$E_{f r a g}^{r e m}$ and $E_{2}$ being the energies of the fragmentation remnant and the additional parton (see fig. 2). We shall again evaluate $E_{\gamma} d^{3} \sigma_{f r a g}^{\text {sub }} / d^{3} p_{\gamma}$ in the small-cone approximation which essentially means that particle ' 2 ' is almost collinear with particle ' 1 ' in fig. 2 . In this approximation we have according to eq. (12)

$$
E_{2}=E_{1} \frac{v(1-w)}{1-v+v w}
$$


where $E_{1}=E_{\gamma} / z$ is the energy of the fragmenting parton before the fragmentation process takes place (see fig. 2). Furthermore, we have

$$
E_{\text {frag }}^{r e m}=(1-z) E_{1}=\frac{1-z}{z} E_{\gamma}
$$

Eq. (30) then turns into

$$
z(1+\epsilon) \leq \frac{1}{1-v+v w}
$$

We are now in the position to write down the general expressions for $E_{\gamma} d^{3} \sigma_{\text {frag }}^{z \geq 1 /(1+\epsilon)} / d^{3} p_{\gamma}$ and $E_{\gamma} d^{3} \sigma_{\text {frag }}^{\text {sub }} / d^{3} p_{\gamma}$ :

$$
\begin{aligned}
E_{\gamma} \frac{d^{3} \sigma_{f r a g}^{z \geq 1 /(1+c)}}{d^{3} p_{\gamma}} & =\frac{1}{\pi p_{T}^{2} S} \sum_{a b c} \int_{\max \left(1-V+V W, \frac{1}{1+\epsilon}\right)}^{1} d z \int_{V W / z}^{1-(1-V) / z} d v \int_{V W / z v}^{1} d w \\
& \times f_{a}\left(x_{1}, M^{2}\right) f_{b}\left(x_{2}, M^{2}\right) D_{c}^{\gamma}\left(z, M_{f}^{2}\right) \hat{s} \frac{d \hat{\sigma}^{a b \rightarrow c}}{d v d w} \\
E_{\gamma} \frac{d^{3} \sigma_{f r a g}^{s u b}}{d^{3} p_{\gamma}} & =\frac{1}{\pi p_{T}^{2} S} \sum_{a b c} \int_{\max \left(1-V+V W, \frac{1}{1+\epsilon}\right)}^{1} d z \int_{V W / z}^{1-(1-V) / z} d v \int_{V W / z v}^{1} d w \\
& \times f_{a}\left(x_{1}, M^{2}\right) f_{b}\left(x_{2}, M^{2}\right) D_{c}^{\gamma}\left(z, M_{f}^{2}\right) \hat{s} \frac{d \hat{\sigma}_{\delta}^{a b \rightarrow c}}{d v d w} \Theta\left(\frac{1}{1-v+v w}-z(1+\epsilon)\right)
\end{aligned}
$$

where $V$ and $W$ are defined as in eq. (2) and

$$
\begin{aligned}
& x_{1}=\frac{V W}{z v w}, \\
& x_{2}=\frac{1-V}{z(1-v)} .
\end{aligned}
$$

The subprocess cross sections $d \hat{\sigma}^{a b \rightarrow c} / d v d w$ for the various contributing $2 \rightarrow 3$ QCD subprocesses with particle $c$ being observed, needed for the calculation of $E_{\gamma} d^{3} \sigma_{f r a g}^{z \geq 1 /(1+\epsilon)} / d^{3} p_{\gamma}$, have been calculated by Aversa et al. [29] and are available in a FORTRAN code. They are of course factorized and thus free of singularities and perturbatively well-defined just as $E_{\gamma} d^{3} \sigma_{d i r}^{i n c l} / d^{3} p_{\gamma}$ in eq. (7) is. We shall now calculate the subprocess cross sections $d \hat{\sigma}_{\delta}^{a b \rightarrow c} / d v d w$ for the subtraction piece in the small-cone approximation. Let us first state what kind of terms are expected in the final result. The first thing to note is that $E_{\gamma} d^{3} \sigma_{\text {frag }}^{i s o l} / d^{3} p_{\gamma}$ as well as $E_{\gamma} d^{3} \sigma_{\text {frag }}^{z \geq 1 /(1+\epsilon)} / d^{3} p_{\gamma}$ and $E_{\gamma} d^{3} \sigma_{\text {frag }}^{\text {sub }} / d^{3} p_{\gamma}$ vanish for $\epsilon \rightarrow 0$ due to the constraint $z \geq 1 /(1+\epsilon)$. For small $\epsilon$ the fragmentation contribution is therefore suppressed proportional to $\epsilon$. In comparison to eq. (9), the basic structure of $E_{\gamma} d^{3} \sigma_{f r a g}^{s u b} / d^{3} p_{\gamma}$ is for small $\epsilon$ and $\delta$ given by

$$
\epsilon\left[\ln \delta\left(\mathcal{A}+\mathcal{A}^{\prime} \ln \epsilon\right)+\mathcal{B}+\mathcal{C} \delta^{2} \ln \epsilon\right]
$$


with new coefficients $\mathcal{A}, \mathcal{A}^{\prime}, \mathcal{B}$ and $\mathcal{C}$. We have anticipated the presence of an $\epsilon \ln \delta \ln \epsilon$ contribution in eq. (37). We shall restrict ourselves to the extraction of the coefficients $\mathcal{A}, \mathcal{A}^{\prime}$ and $\mathcal{B}$ and neglect the contribution from $\mathcal{C}$ which should be less important. This view is supported by the fact that the term $\mathcal{C} \delta^{2} \ln \epsilon$ in eq. (9) also turns out to be numerically rather small. Therefore the neglect of the $\epsilon \delta^{2} \ln \epsilon$ terms here [30] (which are rather hard to calculate) might slightly influence the accuracy of $E_{\gamma} d^{3} \sigma_{f r a g}^{\text {sub }} / d^{3} p_{\gamma}$, but will be completely immaterial for the full final cross section for isolated prompt photon production. The remaining contributions corresponding to the coefficients $\mathcal{A}, \mathcal{A}^{\prime}$ and $\mathcal{B}$ are quite easy to calculate. The principal way to extract these is to take the matrix elements for the $2 \rightarrow 3$ QCD subprocesses (with all possible choices for the observed final state parton) published in [31], impose on them the collinear kinematics worked out in the last subsection and integrate them over phase space via eqs. (10) and (13). In this way one encounters final state collinear $(1 / \tau)$ singularities which are removed by factorization. The justification for this is similar to the discussion accompanying eq. (8): Before the factorization of final state mass singularities has taken place, the pole structure of $E_{\gamma} d^{3} \sigma_{f r a g}^{i s o l} / d^{3} p_{\gamma}=E_{\gamma} d^{3} \sigma_{f r a g}^{z \geq 1 /(1+\varepsilon)} / d^{3} p_{\gamma}-E_{\gamma} d^{3} \sigma_{f r a g}^{s u b} / d^{3} p_{\gamma}$ is schematically given by

$$
\begin{aligned}
\left.E_{\gamma} \frac{d^{3} \sigma_{f r a g}^{i s o l}}{d^{3} p_{\gamma}}\right|_{p o l e} & =\left.E_{\gamma} \frac{d^{3} \sigma_{\text {frag }}^{z \geq 1 /(1+\varepsilon)}}{d^{3} p_{\gamma}}\right|_{\text {pole }}-\left.E_{\gamma} \frac{d^{3} \sigma_{\text {frag }}^{\text {sub }}}{d^{3} p_{\gamma}}\right|_{\text {pole }} \\
& \sim-\frac{\epsilon}{\tau} \mu^{2 \tau} \sum_{a b c d e} \frac{d \hat{\sigma}^{a b \rightarrow c d}}{d v} \otimes f_{a}\left(x_{1}, M^{2}\right) f_{b}\left(x_{2}, M^{2}\right) \otimes \alpha_{s} P_{e c}\left(z^{\prime}\right) \otimes D_{e}^{\gamma}\left(z, M_{f}^{2}\right),
\end{aligned}
$$

a typical graphical representation given by fig. 3. The contribution from (38) is absorbed in (1), leading again to a next-to-leading order fragmentation function $D_{c}^{\gamma}\left(z, M_{f}^{2}\right)$. In our present calculation we can therefore just subtract the poles since those in $E_{\gamma} d^{3} \sigma_{\text {frag }}^{z \geq 1 /(1+\varepsilon)} / d^{3} p_{\gamma}$ (for which we take the results of Aversa et al. [29]) are also already factored out. We only have to make sure that we use the same factorization scheme as that in ref. [29]. In the corresponding FORTRAN code there is an option to choose the $\overline{\mathrm{MS}}$-scheme. We therefore calculate our fragmentation subtraction cross section also in the $\overline{\mathrm{MS}}$-scheme just as we did for the direct piece in the last subsection. Taking a look at eqs. (14)-(18) and the simple structure of eq. (22) emerging from these equations, it is now rather straightforward to obtain the final result. One has to collect all suitable combinations of splitting functions times $2 \rightarrow 2$ QCD cross sections. As before, the $\ln \delta$ pieces (corresponding to coefficients $\left.\mathcal{A}, \mathcal{A}^{\prime}\right)$ are remnants of the factorized collinear singularities and thus have the same structure as the $1 / \tau$ pole terms did. In the $\overline{\mathrm{MS}}$-scheme the non-logarithmic pieces (coefficient $\mathcal{B})$ can again only stem from the $\tau$-terms in the $n$-dimensional splitting functions. Since 
we have in $n=4-2 \tau$ dimensions for $z<1[5]$

$$
\begin{aligned}
& \tilde{P}_{q q}(z, \tau)=C_{F}\left[\frac{1+z^{2}}{1-z}-\tau(1-z)\right] \\
& \tilde{P}_{q g}(z, \tau)=\frac{1}{2(1-\tau)}\left[z^{2}+(1-z)^{2}-\tau\right] \\
& \tilde{P}_{g q}(z, \tau)=C_{F}\left[\frac{1+(1-z)^{2}}{z}-\tau z\right] \\
& \tilde{P}_{g g}(z, \tau)=2 N_{C}\left[\frac{z}{1-z}+\frac{1-z}{z}+z(1-z)\right],
\end{aligned}
$$

we are led to the functions (cf. eqs. (15) and (18))

$$
\begin{aligned}
& \mathcal{P}_{q q}(z)=C_{F}\left[\frac{1+z^{2}}{1-z} \ln \left(\frac{v^{2}(1-w)^{2} E_{1}^{2} \delta^{2}}{M_{f}^{2}}\right)+(1-z)\right] \\
& \mathcal{P}_{q g}(z)=\left[\frac{z^{2}+(1-z)^{2}}{2} \ln \left(\frac{v^{2}(1-w)^{2} E_{1}^{2} \delta^{2}}{M_{f}^{2}}\right)+z(1-z)\right] \\
& \mathcal{P}_{g q}(z)=C_{F}\left[\frac{1+(1-z)^{2}}{z} \ln \left(\frac{v^{2}(1-w)^{2} E_{1}^{2} \delta^{2}}{M_{f}^{2}}\right)+z\right] \\
& \mathcal{P}_{g g}(z)=2 N_{C}\left[\frac{z}{1-z}+\frac{1-z}{z}+z(1-z)\right] \ln \left(\frac{v^{2}(1-w)^{2} E_{1}^{2} \delta^{2}}{M_{f}^{2}}\right),
\end{aligned}
$$

(where $E_{1}=E_{\gamma} / z$ ) which will apear in the final result. Considering, for example, the process $q g \rightarrow g X \rightarrow \gamma X^{\prime}$, the final state gluon fragmenting into the photon, one has (cf. eq. (22))

$\frac{d \hat{\sigma}_{\delta}^{g g \rightarrow g}}{d v d w}=\frac{\alpha_{s}}{2 \pi} \frac{v}{1-v+v w}\left[\mathcal{P}_{g g}(1-v+v w) \frac{d \hat{\sigma}^{q g \rightarrow g q}}{d y}(\hat{s}, y)+\mathcal{P}_{g q}(1-v+v w) \frac{d \hat{\sigma}^{q g \rightarrow q g}}{d y}(\hat{s}, y)\right]$

to be used in eq. (35) with $y=v w /(1-v+v w)$. The two terms in eq. (41) correpsond to figs. $4 \mathrm{a}$ and $4 \mathrm{~b}$. One observes that the diagonal splitting function $\mathcal{P}_{g g}(z)$ appears in the result which produces a $1 /(1-w)$ term, for $\epsilon \rightarrow 0$ ultimately giving rise to $\epsilon \ln \delta \ln \epsilon$ terms as promised in eq. (37). Since the structure of the final results is very simple it is possible to write down the $d \hat{\sigma}_{\delta}^{a b \rightarrow c} / d v d w$ for all subprocesses without using the $2 \rightarrow 3$ matrix elements calculated in [31] at all. As a check we have used these matrix elements for several subprocesses and integrated them in the small-cone approximation. After $\overline{\mathrm{MS}}$ factorization the final result always turned out to be the same. We collect the results for all subprocesses in appendix B [27]. 


\subsection{Isolated prompt photon production with longitudinally po- larized beams}

In this subsection we want to briefly show how our results need to be modified in order to deal with longitudinal polarization of the incoming hadrons. This issue should become relevant at future high-energy colliders such as RHIC where it will be possible to perform experiments with two colliding longitudinally polarized hadron beams. Only a few replacements are necessary in our previous formulas to make them suitable for the polarized case. The quantity of interest now is the cross section difference

$$
E_{\gamma} \frac{d^{3} \Delta \sigma}{d^{3} p_{\gamma}} \equiv E_{\gamma} \frac{d^{3} \sigma(++)}{d^{3} p_{\gamma}}-E_{\gamma} \frac{d^{3} \sigma(+-)}{d^{3} p_{\gamma}},
$$

$E_{\gamma} d^{3} \sigma\left(h_{1} h_{2}\right) / d^{3} p_{\gamma}$ denoting the cross section for the production of a prompt photon by incoming hadrons with helicities $h_{1}$ and $h_{2}$. Let us also introduce the polarized parton distributions

$$
\Delta f_{a}^{A}\left(x, M^{2}\right) \equiv\left(f_{a}^{A}\right)_{+}\left(x, M^{2}\right)-\left(f_{a}^{A}\right)_{-}\left(x, M^{2}\right),
$$

where $\left(f_{a}^{A}\right)_{+(-)}$denotes the distribution of parton type $a$ with positive (negative) helicity in hadron $A$ with positive helicity. It should be noted that taking the sum instead of the difference in eqs. (42) and (43) one recovers the usual unpolarized quantities $E_{\gamma} d^{3} \sigma / d^{3} p_{\gamma}$ and $f_{a}^{A}\left(x, M^{2}\right)$ introduced before. We can now easily adapt the cross sections in eqs. (1), (19), (22), (25), (26), (28), (34) and (35) to the polarized case by making the replacements

$$
\begin{aligned}
f_{a}^{A}\left(x, M^{2}\right) & \longrightarrow \Delta f_{a}^{A}\left(x, M^{2}\right) \\
\text { and } \quad \sigma & \longrightarrow \Delta \sigma
\end{aligned}
$$

for all parton distributions and cross sections. The latter replacement corresponds to hadronic as well as subprocess cross sections, the ' $\Delta$ ' being defined as in eq. (42) as the difference of cross sections for the two different relative settings of the incoming particles' helicities. Analogously, we have to replace $T_{q \bar{q}}$ and $T_{q g}$ in eq. (27) by

$$
\begin{aligned}
T_{q \bar{q}} \longrightarrow \Delta T_{q \bar{q}} & =-T_{q \bar{q}}=-\left(v^{2}+(1-v)^{2}\right) \\
T_{q g} \longrightarrow \Delta T_{q g} & =1-(1-v)^{2} .
\end{aligned}
$$

Note, however, that we must not change the photon fragmentation function $D_{c}^{\gamma}\left(z, M_{f}^{2}\right)$ in our previous equations since we are dealing with unpolarized prompt photons and only the initial state is polarized. The same is true for the functions $\mathcal{P}_{\gamma q}(z)$ in eq. (18) and 
$\mathcal{P}_{i j}(i, j=q, g)$ in eq. (40). These functions also refer to the final state and thus have to be kept.

The subprocess cross sections $d \Delta \hat{\sigma}^{a b} / d v d w$ for the direct part of inclusive polarized prompt photon production needed in the polarized versions of eqs. (7) and (28) were recently calculated in refs. $[12,13]$ in the $\overline{\mathrm{MS}}$-scheme. In the latter calculation [13] the (consistent) HVBM-scheme [32] was used to deal with $\gamma_{5}$ and $\epsilon_{\mu \nu \rho \sigma}$ in $n=4-2 \tau$ dimensions. Making the modifications (44) and (45) in eqs. (19), (22) and (25)-(27) now enables us to perform isolation cuts also on the direct piece of the polarized prompt photon cross section. We present the $d \Delta \hat{\sigma}^{a b \rightarrow c d}(\hat{s}, y) / d y$ (which we now need) in appendix C. Let us note that our results do not depend on the $\gamma_{5}$ scheme chosen because the function $\mathcal{P}_{\gamma q}(z)$ in eq. (18) does not, since it is unpolarized.

Unfortunately, the next-to-leading order fragmentation contribution can not yet be calculated for the polarized case. The reason is that the cross sections for all the polarized $2 \rightarrow 3$ QCD subprocesses are not known up to now [33]. Nevertheless, it is straightforward by using the results of appendix $\mathrm{C}$ to obtain the polarized subtraction piece subprocess cross sections $d \Delta \hat{\sigma}_{\delta}^{a b \rightarrow c} / d v d w$ (to be used in the polarized version of eq. (35)). Again, these results do not depend on the treatment of $\gamma_{5}$. They will become relevant when the other ingredient of $E_{\gamma} d^{3} \Delta \sigma_{f r a g}^{i s o l} / d^{3} p_{\gamma}$, namely $E_{\gamma} d^{3} \Delta \sigma_{f r a g}^{z \geq 1 /(1+c)} / d^{3} p_{\gamma}$ (cf. eq. (29)), can one day be calculated.

\section{Numerical results}

We shall now present some numerical results for our approximation method for unpolarized isolated prompt photon production. It is not the purpose of this study to make quantitative comparisons with existing collider data on isolated prompt photon production $[16,17]$. Rather, we want to test the accuracy of our method and show the general size of the isolation effects for the direct and the fragmentation contribution in next-to-leading order. We reserve the actual comparison with data for a future publication [34].

The first thing we want to study is the accuracy of our approximation method for the direct contribution to isolated prompt photon production. For this purpose we have to compare with the 'exact' numerical solution from a Monte Carlo program. Since the program of Baer et al. [5] is not freely distributed we have set up our own Monte Carlo 
code which calculates the subtraction piece $E_{\gamma} d^{3} \sigma_{d i r}^{s u b} / d^{3} p_{\gamma}$ in eq. (7). For this program we have used our own results [13] for the unintegrated $2 \rightarrow 3$ matrix elements for $a b \rightarrow \gamma c d$. The actual Monte Carlo calculation is performed following the lines outlined in ref. [5]. We have assumed a very small cone around the photon with opening $\delta_{c} \ll \delta$ which is located inside the isolation cone and is concentric to it. Inside the very small cone we can safely calculate everything analytically as outlined in the last section and can perform the $\overline{\mathrm{MS}}$ factorization of final state mass singularities [35]. For the rest of the calculation we can now use the matrix elements in four dimensions and numerically integrate them over phase space, taking care of the proper isolation constraints. We have checked that the final Monte Carlo results are independent of the collinear cut-off $\delta_{c}$ over a wide range of $\delta_{c}$ values, as they must be.

For our numerical evaluation of $E_{\gamma} d^{3} \sigma_{d i r}^{s u b} / d^{3} p_{\gamma}$ we use the GRV parton distributions in next-to-leading order [36] which have been found to be in perfect agreement with recent HERA data for $F_{2}^{p}[37,38]$. For consistency we calculate the strong coupling $\alpha_{s}\left(\mu^{2}\right)$ ( $\mu$ being the renormalization scale) using the two loop expression for it and taking the $\Lambda_{Q C D}$ values and threshold conventions determined along with the structure functions in ref. [36]. We perform our calculations by setting $N_{f}=5$, neglecting, however, the contributions from $b$ quarks. We study the case of $p \bar{p}$ collisions at $\sqrt{S}=1 \mathrm{TeV}$ which is a typical value for recent $p \bar{p}$ colliders. We choose $\mu=M=p_{T}$ for the renormalization and (initial state) factorization scales and $M_{f}=\delta p_{T}$ for the fragmentation scale unless otherwise stated. In figs. $5 \mathrm{a}, \mathrm{b}$ we show the relative deviation

$$
D \equiv \frac{\left(E_{\gamma} d^{3} \sigma_{d i r}^{s u b} / d^{3} p_{\gamma}\right)_{M C}-\left(E_{\gamma} d^{3} \sigma_{d i r}^{s u b} / d^{3} p_{\gamma}\right)_{a p p}}{\left(E_{\gamma} d^{3} \sigma_{d i r}^{s u b} / d^{3} p_{\gamma}\right)_{M C}}
$$

as a function of $\delta$ and $\epsilon$ for two values of the transverse momentum, $p_{T}=20 \mathrm{GeV}$ and $p_{T}=50 \mathrm{GeV}$, at zero rapidity, $\eta=0$. One can easily see that the accuracy of our approximation for the subtraction cross section $E_{\gamma} d^{3} \sigma_{d i r}^{s u b} / d^{3} p_{\gamma}$ is much better than $10 \%$ over a wide range of values for $\delta$ and $\epsilon$. It should be emphasized that the subtraction cross section is only a subdominant part of the full isolated cross section (see below). Therefore our approximation for $E_{\gamma} d^{3} \sigma_{d i r}^{i s o l} / d^{3} p_{\gamma}$ is generally far better than $10 \%$. According to figs. 5 a,b the approximation for $E_{\gamma} d^{3} \sigma_{d i r}^{s u b} / d^{3} p_{\gamma}$ tends to break down at large $\epsilon$ and, in particular, at very large $\delta$ which of course is expected. Nevertheless, even for $\delta=0.7$ and $\epsilon=0.2$ it is still very good. It should be stressed that the Monte Carlo program is very computertime consuming [39] and has fluctuations which are sometimes even of the order of the accuracy of our approximation method. This can be seen in figs. $5 \mathrm{a}, \mathrm{b}$ which have not 
been smoothed. For these reasons our approximation is very convenient.

At larger $p_{T}$ the approximation seems to become slightly worse. This is, however, no drawback since the subtraction piece becomes rather small and eventually negligible at large $p_{T}$. This is shown in fig. 6 where we have plotted the ratio

$$
R_{d i r} \equiv-\frac{E_{\gamma} d^{3} \sigma_{d i r}^{s u b} / d^{3} p_{\gamma}}{E_{\gamma} d^{3} \sigma_{d i r}^{i s o l} / d^{3} p_{\gamma}}=-\frac{E_{\gamma} d^{3} \sigma_{d i r}^{s u b} / d^{3} p_{\gamma}}{E_{\gamma} d^{3} \sigma_{d i r}^{i n c l} / d^{3} p_{\gamma}-E_{\gamma} d^{3} \sigma_{d i r}^{s u b} / d^{3} p_{\gamma}}
$$

vs. $p_{T}$ for fixed $\delta=0.4$ and $\epsilon=0.1$. We have calculated $R_{d i r}$ using our approximation method for $E_{\gamma} d^{3} \sigma_{d i r}^{s u b} / d^{3} p_{\gamma}$ (solid line) and also using our Monte Carlo program (dashed line). As can be seen, both results are again very similar. The ratio $R_{d i r}$ has decreased to less than $5 \%$ already at $p_{T} \approx 50 \mathrm{GeV}$. An error of roughly $10 \%$ in our approximation for $E_{\gamma} d^{3} \sigma_{d i r}^{s u b} / d^{3} p_{\gamma}$ at this $p_{T}$ thus leads to an overall error in $E_{\gamma} d^{3} \sigma_{d i r}^{i s o l} / d^{3} p_{\gamma}$ of about $0.5 \%$ which is certainly completely negligible. Note, however, that $E_{\gamma} d^{3} \sigma_{d i r}^{s u b} / d^{3} p_{\gamma}$ is important at small $p_{T}$, exactly where our approximation is most accurate. From fig. 6 one infers that $E_{\gamma} d^{3} \sigma_{d i r}^{s u b} / d^{3} p_{\gamma}$ is negative. This feature stems from the logarithm in eq. (18) which gives a negative contribution. The effect of subtracting $E_{\gamma} d^{3} \sigma_{d i r}^{s u b} / d^{3} p_{\gamma}$ from $E_{\gamma} d^{3} \sigma_{d i r}^{i n c l} / d^{3} p_{\gamma}$ is thus to increase the cross section.

Let us note that our approximation breaks down at large rapidities. According to the jet study of ref. [40], $\delta \cosh \eta$, not $\delta$, is the relevant quantity that must be small for the small-cone approximation to be a good approximation. Thus at large $\eta, \delta$ must be very small in order to make $\delta \cosh \eta$ still small. We have found this criterion to be confirmed by our results. At rapidity $\eta=1$ our approximation starts to break down already at $\delta=0.6$

The success of our approximation method for the direct piece implies that our approximation also works for the next-to-leading order fragmentation contribution. We do not attempt to check the accuracy of our approximation for this contribution numerically by a Monte Carlo program since this is a rather hard task and needless in view of our results for the direct piece. From now on we shall solely use our approximations for the subtraction cross sections.

We now show some results for the effects of isolation on the next-to-leading order fragmentation contribution. In addition to the distributions and parameters used before, we use the next-to-leading order photon fragmentation functions of GRV [41] which we transform from the $\mathrm{DIS}_{\gamma}$-scheme to the $\overline{\mathrm{MS}}$-scheme as described in ref. [41]. For the 
calculation of $E_{\gamma} d^{3} \sigma_{f r a g}^{z \geq 1 /(1+\varepsilon)} / d^{3} p_{\gamma}$ we use the program of [29]. Fig. 7 shows the ratio (cf. eq. (29))

$$
R_{f r a g} \equiv-\frac{E_{\gamma} d^{3} \sigma_{f r a g}^{s u b} / d^{3} p_{\gamma}}{E_{\gamma} d^{3} \sigma_{f r a g}^{i s o l} / d^{3} p_{\gamma}}=-\frac{E_{\gamma} d^{3} \sigma_{f r a g}^{s u b} / d^{3} p_{\gamma}}{E_{\gamma} d^{3} \sigma_{f r a g}^{z \geq 1 /(1+\epsilon)} / d^{3} p_{\gamma}-E_{\gamma} d^{3} \sigma_{f r a g}^{\text {sub }} / d^{3} p_{\gamma}}
$$

versus $p_{T}$ for $\delta=0.4$ and $\epsilon=0.1$. It becomes obvious that the influence of the subtraction piece is again small at large $p_{T}$ but important at small $p_{T}$. As for the direct case, the subtraction piece is again negative, thus enhancing the cross section. From figs. 6 and 7 one also infers that $R_{f r a g}>R_{d i r}$, i.e. in the case of fragmentation the subtraction piece is more important relative to the cross section it is subtracted from. It should, however, be noted that the full isolated fragmentation cross section $E_{\gamma} d^{3} \sigma_{\text {frag }}^{i s o l} / d^{3} p_{\gamma}$ is strongly reduced by the constraint (4) and much smaller than it would be without any isolation cut. To study its importance, fig. 8 shows the ratio

$$
\begin{aligned}
R & \equiv \frac{E_{\gamma} d^{3} \sigma_{\text {frag }}^{i s o l} / d^{3} p_{\gamma}}{E_{\gamma} d^{3} \sigma_{d i r}^{\text {isol }} / d^{3} p_{\gamma}+E_{\gamma} d^{3} \sigma_{\text {frag }}^{\text {isol }} / d^{3} p_{\gamma}} \\
& =\frac{E_{\gamma} d^{3} \sigma_{\text {frag }}^{z \geq 1 /(1+c)} / d^{3} p_{\gamma}-E_{\gamma} d^{3} \sigma_{f r a g}^{\text {sub }} / d^{3} p_{\gamma}}{E_{\gamma} d^{3} \sigma_{d i r}^{\text {incl }} / d^{3} p_{\gamma}-E_{\gamma} d^{3} \sigma_{\text {dir }}^{\text {sub }} / d^{3} p_{\gamma}+E_{\gamma} d^{3} \sigma_{\text {frag }}^{z \geq 1 /(1+\epsilon)} / d^{3} p_{\gamma}-E_{\gamma} d^{3} \sigma_{f r a g}^{\text {sub }} / d^{3} p_{\gamma}}
\end{aligned}
$$

as a function of $p_{T}$ for the above values for $\delta$ and $\epsilon$. Fig. 8 shows that the influence of the isolated next-to-leading order fragmentation contribution on the full cross section for isolated prompt photon production is substantial at small $p_{T}$ but not dominant.

In figs. 9 and 10 we show our results for the dependence of $E_{\gamma} d^{3} \sigma_{d i r}^{i s o l} / d^{3} p_{\gamma}+E_{\gamma} d^{3} \sigma_{f r a g}^{i s o l} / d^{3} p_{\gamma}$ on the parameters $\epsilon$ and $\delta$, which was also calculated in ref. [6] where the program of Baer et al. [5] was used. We use three different values for $p_{T}, p_{T}=20,50$ and $100 \mathrm{GeV}$ at fixed $\delta=0.4$ (for fig. 9) or fixed $\epsilon=0.1$ (for fig. 10). We can reproduce the rather flat behavior found in [6] which once more demonstrates the correctness of our results.

Finally we briefly study the dependence of our results on the choice for the fragmentation scale $M_{f}$. As we discussed in subsection 2.1, the dependence on the fragmentation scale would drop out completely from $E_{\gamma} d^{3} \sigma_{d i r}^{i s o l} / d^{3} p_{\gamma}$ (which dominates the full isolated cross section) if it were possible to set $\epsilon=0$ there. For $\epsilon \neq 0$ but small we thus anticipate a very weak dependence on $M_{f}$. This can be seen in fig. 11 where we show the full isolated cross section for prompt photon production (i.e. $E_{\gamma} d^{3} \sigma_{d i r}^{i s o l} / d^{3} p_{\gamma}+E_{\gamma} d^{3} \sigma_{f r a g}^{\text {isol }} / d^{3} p_{\gamma}$ ) in next-to-leading order as a function of $M_{f}$ for two fixed values of $p_{T}$ and fixed $\mu=M=p_{T}$, using again $\delta=0.4$ and $\epsilon=0.1$. The result is obviously a straight line over a wide range of $M_{f}$ values. 
As mentioned above, we reserve a comparison with existing collider data $[16,17]$ on isolated prompt photon production to a forthcoming publication [34].

\section{Acknowledgements}

We are grateful to M. Glück and E. Reya for stimulating our interest in this problem and for many helpful discussions. We are also thankful to E. Reya for carefully reading the manuscript. We are furthermore thankful to J.Ph. Guillet for providing us with the FORTRAN code for next-to-leading order inclusive hadron production, and for helpful explanations. We thank A. Vogt for his help with the FORTRAN code for the evolution of the next-to-leading order fragmentation functions of ref. [41]. This work has been supported in part by the 'Bundesministerium für Forschung und Technologie', Bonn. 


\section{Appendix A: $2 \rightarrow 2$ QCD cross sections}

In this appendix we collect the unpolarized $2 \rightarrow 2$ QCD cross sections $d \hat{\sigma}^{a b \rightarrow c d}(\hat{s}, y) / d y$ for the processes $a b \rightarrow c d$ (see for example [42]), to be used in eq. (22) and in our analytical results for the fragmentation subtraction cross section in appendix $B$. Using $\hat{t} \equiv\left(p_{a}-p_{c}\right)^{2}=-\hat{s}(1-y)$ and $\hat{u} \equiv\left(p_{b}-p_{c}\right)^{2}=-\hat{s} y$ we have:

$$
\begin{aligned}
& \frac{d \hat{\sigma}^{q q^{\prime} \rightarrow q q^{\prime}}}{d y}(\hat{s}, y)=\frac{C_{F}}{N_{C}} \frac{\pi \alpha_{s}^{2}}{\hat{s}} \frac{\hat{s}^{2}+\hat{u}^{2}}{\hat{t}^{2}} \\
& \frac{d \hat{\sigma}^{q \bar{q} \rightarrow q^{\prime} \bar{q}^{\prime}}}{d y}(\hat{s}, y)=\frac{C_{F}}{N_{C}} \frac{\pi \alpha_{s}^{2}}{\hat{s}} \frac{\hat{t}^{2}+\hat{u}^{2}}{\hat{s}^{2}} \\
& \frac{d \hat{\sigma}^{q q \rightarrow q q}}{d y}(\hat{s}, y)=\frac{C_{F}}{N_{C}} \frac{\pi \alpha_{s}^{2}}{\hat{s}}\left[\frac{\hat{s}^{2}+\hat{u}^{2}}{\hat{t}^{2}}+\frac{\hat{s}^{2}+\hat{t}^{2}}{\hat{u}^{2}}-\frac{2}{N_{C}} \frac{\hat{s}^{2}}{\hat{t} \hat{u}}\right] \\
& \frac{d \hat{\sigma}^{q \bar{q} \rightarrow q \bar{q}}}{d y}(\hat{s}, y)=\frac{C_{F}}{N_{C}} \frac{\pi \alpha_{s}^{2}}{\hat{s}}\left[\frac{\hat{s}^{2}+\hat{u}^{2}}{\hat{t}^{2}}+\frac{\hat{t}^{2}+\hat{u}^{2}}{\hat{s}^{2}}-\frac{2}{N_{C}} \frac{\hat{u}^{2}}{\hat{s} \hat{t}}\right] \\
& \frac{d \hat{\sigma}^{q g \rightarrow q g}}{d y}(\hat{s}, y)=\frac{1}{N_{C}} \frac{\pi \alpha_{s}^{2}}{\hat{s}}\left(\hat{s}^{2}+\hat{u}^{2}\right)\left[\frac{N_{C}}{\hat{t}^{2}}-\frac{C_{F}}{\hat{s} \hat{u}}\right] \\
& \frac{d \hat{\sigma}^{q \bar{q} \rightarrow g g}}{d y}(\hat{s}, y)=\frac{2 C_{F}}{N_{C}} \frac{\pi \alpha_{s}^{2}}{\hat{s}}\left(\hat{t}^{2}+\hat{u}^{2}\right)\left[\frac{C_{F}}{\hat{t} \hat{u}}-\frac{N_{C}}{\hat{s}^{2}}\right] \\
& \frac{d \hat{\sigma}^{g g \rightarrow q \bar{q}}}{d y}(\hat{s}, y)=\frac{1}{2 C_{F} N_{C}} \frac{\pi \alpha_{s}^{2}}{\hat{s}}\left(\hat{t}^{2}+\hat{u}^{2}\right)\left[\frac{C_{F}}{\hat{t} \hat{u}}-\frac{N_{C}}{\hat{s}^{2}}\right] \\
& \frac{d \hat{\sigma}^{g g \rightarrow g g}}{d y}(\hat{s}, y)=\frac{N_{C}}{2 C_{F}} \frac{\pi \alpha_{s}^{2}}{\hat{s}} \frac{\left(\hat{s}^{4}+\hat{t}^{4}+\hat{u}^{4}\right)}{\hat{s}^{2} \hat{t}^{2} \hat{u}^{2}}\left(\hat{s}^{2}+\hat{t}^{2}+\hat{u}^{2}\right)
\end{aligned}
$$

where $C_{F}=4 / 3, N_{C}=3$, and $N_{f}$ is the number of active flavors.

\section{Appendix B: Final results for the fragmentation sub- traction cross section}

In this appendix we present the cross sections $d \hat{\sigma}_{\delta}^{a b \rightarrow c} / d v d w$ (to be used in eq. (35)) for the various processes $a b \rightarrow c X$, where parton $c$ fragments into the photon. We order the processes in the same way as it was done in the FORTRAN code of ref. [29]. Defining the common factor

$$
\mathcal{N} \equiv \frac{\alpha_{s}}{2 \pi} \frac{v}{1-v+v w}
$$

and using the functions $\mathcal{P}_{i j}(i, j=q, g)$ defined in eq. (40), we have [27]: 


$$
\begin{aligned}
& \frac{d \hat{\sigma}_{\delta}^{q q^{\prime} \rightarrow q}}{d v d w}=\mathcal{N} \mathcal{P}_{q q}(z) \frac{d \hat{\sigma}^{q q^{\prime} \rightarrow q q^{\prime}}}{d y}(\hat{s}, y) \\
& \frac{d \hat{\sigma}_{\delta}^{q q^{\prime} \rightarrow g}}{d v d w}=\mathcal{N}\left(\mathcal{P}_{g q}(z) \frac{d \hat{\sigma}^{q q^{\prime} \rightarrow q q^{\prime}}}{d y}(\hat{s}, y)+\mathcal{P}_{g q}(z) \frac{d \hat{\sigma}^{q q^{\prime} \rightarrow q^{\prime} q}}{d y}(\hat{s}, y)\right) \\
& \frac{d \hat{\sigma}_{\delta}^{q \bar{q}^{\prime} \rightarrow q}}{d v d w}=\mathcal{N} \mathcal{P}_{q q}(z) \frac{d \hat{\sigma}^{q q^{\prime} \rightarrow q q^{\prime}}}{d y}(\hat{s}, y) \\
& \frac{d \hat{\sigma}_{\delta}^{q \bar{q}^{\prime} \rightarrow g}}{d v d w}=\mathcal{N}\left(\mathcal{P}_{g q}(z) \frac{d \hat{\sigma}^{q q^{\prime} \rightarrow q q^{\prime}}}{d y}(\hat{s}, y)+\mathcal{P}_{g q}(z) \frac{d \hat{\sigma}^{q q^{\prime} \rightarrow q^{\prime} q}}{d y}(\hat{s}, y)\right) \\
& \frac{d \hat{\sigma}_{\delta}^{q \bar{q} \rightarrow q^{\prime}}}{d v d w}=\mathcal{N}\left(\mathcal{P}_{q q}(z) \frac{d \hat{\sigma}^{q \bar{q} \rightarrow q^{\prime} \bar{q}^{\prime}}}{d y}(\hat{s}, y)+\mathcal{P}_{q g}(z) \frac{d \hat{\sigma}^{q \bar{q} \rightarrow g g}}{d y}(\hat{s}, y)\right) \\
& \frac{d \hat{\sigma}_{\delta}^{q q \rightarrow q}}{d v d w}=\mathcal{N} \mathcal{P}_{q q}(z) \frac{d \hat{\sigma}^{q q \rightarrow q q}}{d y}(\hat{s}, y) \\
& \frac{d \hat{\sigma}_{\delta}^{q q \rightarrow g}}{d v d w}=\mathcal{N} \mathcal{P}_{g q}(z) \frac{d \hat{\sigma}^{q q \rightarrow q q}}{d y}(\hat{s}, y) \\
& \frac{d \hat{\sigma}_{\delta}^{q g \rightarrow q^{\prime}}}{d v d w}=\mathcal{N} \mathcal{P}_{q g}(z) \frac{d \hat{\sigma}^{q g \rightarrow g q}}{d y}(\hat{s}, y) \\
& \frac{d \hat{\sigma}_{\delta}^{g g \rightarrow \bar{q}^{\prime}}}{d v d w}=\mathcal{N} \mathcal{P}_{q g}(z) \frac{d \hat{\sigma}^{q g \rightarrow g q}}{d y}(\hat{s}, y) \\
& \frac{d \hat{\sigma}_{\delta}^{q g \rightarrow \bar{q}}}{d v d w}=\mathcal{N} \mathcal{P}_{q g}(z) \frac{d \hat{\sigma}^{q g \rightarrow g q}}{d y}(\hat{s}, y) \\
& \frac{d \hat{\sigma}_{\delta}^{q \bar{q} \rightarrow q}}{d v d w}=\mathcal{N}\left(\mathcal{P}_{q g}(z) \frac{d \hat{\sigma}^{q \bar{q} \rightarrow g g}}{d y}(\hat{s}, y)+\mathcal{P}_{q q}(z) \frac{d \hat{\sigma}^{q \bar{q} \rightarrow q \bar{q}}}{d y}(\hat{s}, y)\right) \\
& \frac{d \hat{\sigma}_{\delta}^{q \bar{q} \rightarrow g}}{d v d w}=\mathcal{N}\left(\mathcal{P}_{g g}(z) \frac{d \hat{\sigma}^{q \bar{q} \rightarrow g g}}{d y}(\hat{s}, y)+\mathcal{P}_{g q}(z) \frac{d \hat{\sigma}^{q \bar{q} \rightarrow q \bar{q}}}{d y}(\hat{s}, y)\right. \\
& \left.+\mathcal{P}_{g q}(z) \frac{d \hat{\sigma}^{q \bar{q} \rightarrow \bar{q} q}}{d y}(\hat{s}, y)+2\left(N_{f}-1\right) \mathcal{P}_{g q}(z) \frac{d \hat{\sigma}^{q \hat{q} \rightarrow q^{\prime} \bar{q}^{\prime}}}{d y}(\hat{s}, y)\right) \\
& \frac{d \hat{\sigma}_{\delta}^{q g \rightarrow q}}{d v d w}=\mathcal{N}\left(\mathcal{P}_{q g}(z) \frac{d \hat{\sigma}^{q g \rightarrow g q}}{d y}(\hat{s}, y)+\mathcal{P}_{q q}(z) \frac{d \hat{\sigma}^{q g \rightarrow q g}}{d y}(\hat{s}, y)\right) \\
& \frac{d \hat{\sigma}_{\delta}^{q g \rightarrow g}}{d v d w}=\mathcal{N}\left(\mathcal{P}_{g g}(z) \frac{d \hat{\sigma}^{q g \rightarrow g q}}{d y}(\hat{s}, y)+\mathcal{P}_{g q}(z) \frac{d \hat{\sigma}^{q g \rightarrow q g}}{d y}(\hat{s}, y)\right) \\
& \frac{d \hat{\sigma}_{\delta}^{g g \rightarrow g}}{d v d w}=\mathcal{N}\left(\mathcal{P}_{g g}(z) \frac{d \hat{\sigma}^{g g \rightarrow g g}}{d y}(\hat{s}, y)+2 N_{f} \mathcal{P}_{g q}(z) \frac{d \hat{\sigma}^{g g \rightarrow q \tilde{q}}}{d y}(\hat{s}, y)\right) \\
& \frac{d \hat{\sigma}_{\delta}^{g g \rightarrow q}}{d v d w}=\mathcal{N}\left(\mathcal{P}_{q g}(z) \frac{d \hat{\sigma}^{g g \rightarrow g g}}{d y}(\hat{s}, y)+\mathcal{P}_{q q}(z) \frac{d \hat{\sigma}^{g g \rightarrow q \bar{q}}}{d y}(\hat{s}, y)\right)
\end{aligned}
$$

where $z=1-v+v w$ and $y=v w /(1-v+v w)$, with $d \hat{\sigma}^{a b \rightarrow c d}(\hat{s}, y) / d y$ given in eq. (50). 


\section{Appendix C: Results for the polarized case}

In this appendix we finally collect our results for the case of longitudinal polarization. For this purpose we only need to know the polarized counterparts $d \Delta \hat{\sigma}^{a b \rightarrow c d}(\hat{s}, y) / d y$ of the cross sections $d \hat{\sigma}^{a b \rightarrow c d}(\hat{s}, y) / d y$ presented in appendix A. According to the discussion in section 2.3 we then only have to replace the $d \hat{\sigma}^{a b \rightarrow c d}(\hat{s}, y) / d y$ by the $d \Delta \hat{\sigma}^{a b \rightarrow c d}(\hat{s}, y) / d y$ in the relevant equations (22) and (51) to obtain the polarized subtraction piece subprocess cross sections $d \Delta \hat{\sigma}_{\delta}^{a b} / d v d w$ and $d \Delta \hat{\sigma}_{\delta}^{a b \rightarrow c} / d v d w$. The cross sections $d \Delta \hat{\sigma}^{a b \rightarrow c d}(\hat{s}, y) / d y$ read [42]:

$$
\begin{aligned}
& \frac{d \Delta \hat{\sigma}^{q q^{\prime} \rightarrow q q^{\prime}}}{d y}(\hat{s}, y)=\frac{C_{F}}{N_{C}} \frac{\pi \alpha_{s}^{2}}{\hat{s}} \frac{\hat{s}^{2}-\hat{u}^{2}}{\hat{t}^{2}} \\
& \frac{d \Delta \hat{\sigma}^{q \bar{q} \rightarrow q^{\prime} \bar{q}^{\prime}}}{d y}(\hat{s}, y)=-\frac{C_{F}}{N_{C}} \frac{\pi \alpha_{s}^{2}}{\hat{s}} \frac{\hat{t}^{2}+\hat{u}^{2}}{\hat{s}^{2}} \\
& \frac{d \Delta \hat{\sigma}^{q q \rightarrow q q}}{d y}(\hat{s}, y)=\frac{C_{F}}{N_{C}} \frac{\pi \alpha_{s}^{2}}{\hat{s}}\left[\frac{\hat{s}^{2}-\hat{u}^{2}}{\hat{t}^{2}}+\frac{\hat{s}^{2}-\hat{t}^{2}}{\hat{u}^{2}}-\frac{2}{N_{C}} \frac{\hat{s}^{2}}{\hat{t} \hat{u}}\right] \\
& \frac{d \Delta \hat{\sigma}^{q \bar{q} \rightarrow q \bar{q}}}{d y}(\hat{s}, y)=\frac{C_{F}}{N_{C}} \frac{\pi \alpha_{s}^{2}}{\hat{s}}\left[\frac{\hat{s}^{2}-\hat{u}^{2}}{\hat{t}^{2}}-\frac{\hat{t}^{2}+\hat{u}^{2}}{\hat{s}^{2}}+\frac{2}{N_{C}} \frac{\hat{u}^{2}}{\hat{s} \hat{t}}\right] \\
& \frac{d \Delta \hat{\sigma}^{q g \rightarrow q g}}{d y}(\hat{s}, y)=\frac{1}{N_{C}} \frac{\pi \alpha_{s}^{2}}{\hat{s}}\left(\hat{s}^{2}-\hat{u}^{2}\right)\left[\frac{N_{C}}{\hat{t}^{2}}-\frac{C_{F}}{\hat{s} \hat{u}}\right] \\
& \frac{d \Delta \hat{\sigma}^{q \bar{q} \rightarrow g g}}{d y}(\hat{s}, y)=-\frac{2 C_{F}}{N_{C}} \frac{\pi \alpha_{s}^{2}}{\hat{s}}\left(\hat{t}^{2}+\hat{u}^{2}\right)\left[\frac{C_{F}}{\hat{t} \hat{u}}-\frac{N_{C}}{\hat{s}^{2}}\right] \\
& \frac{d \Delta \hat{\sigma}^{g g \rightarrow q \bar{q}}}{d y}(\hat{s}, y)=-\frac{1}{2 C_{F} N_{C}} \frac{\pi \alpha_{s}^{2}}{\hat{s}^{2}}\left(\hat{t}^{2}+\hat{u}^{2}\right)\left[\frac{C_{F}}{\hat{t} \hat{u}}-\frac{N_{C}}{\hat{s}^{2}}\right] \\
& \frac{d \Delta \hat{\sigma}^{g g \rightarrow g g}}{d y}(\hat{s}, y)=\frac{N_{C}}{2 C_{F}} \frac{\pi \alpha_{s}^{2}}{\hat{s}} \frac{\left(\hat{s}^{4}-\hat{t}^{4}-\hat{u}^{4}\right)}{\hat{s}^{2} \hat{t}^{2} \hat{u}^{2}}\left(\hat{s}^{2}+\hat{t}^{2}+\hat{u}^{2}\right) .
\end{aligned}
$$




\section{References}

[1] H. Fritzsch and P. Minkowski, Phys. Lett. 69B, 316 (1977);

R. Rückl, S. Brodsky and J. Gunion, Phys. Rev. D18, 2469 (1978);

For reviews on the older literature see: T. Ferbel and W. Molzon, Rev. Mod. Phys. 56, 181 (1984) and J.F. Owens, Rev. Mod. Phys. 59, 465 (1987).

[2] P. Aurenche, R. Baier, A. Douiri, M. Fontannaz and D. Schiff, Phys. Lett. 140B, 87 (1984).

[3] P. Aurenche, R. Baier, M. Fontannaz and D. Schiff, Nucl. Phys. B297, 661 (1988).

[4] P. Aurenche, R. Baier, M. Fontannaz, J.F. Owens and M. Werlen, Phys. Rev. D39, 3275 (1989).

[5] H. Baer, J. Ohnemus and J.F. Owens, Phys. Lett. B234, 127 (1990); Phys. Rev. D42, 61 (1990).

[6] E.L. Berger and J. Qiu, Phys. Lett. B248, 371 (1990); Phys. Rev. D44, 2002 (1991).

[7] A.P. Contogouris, S. Papadopoulos and D. Atwood, Theor. Math. Phys. 87, 374 (1991).

[8] J. Ashman et al., EMC Collab., Phys. Lett. B206, 364 (1988); Nucl. Phys. B328, 1 (1989).

[9] B. Adeva et al., SMC Collab., Phys. Lett. B302, 533 (1993).

[10] P.L. Anthony et al., E142 Collab., SLAC report SLAC PUB-6101.

[11] E.L. Berger and J. Qiu, Phys. Rev. D40, 778 (1989); D40, 3128 (1989);

S. Gupta, D. Indumathi and M.V.N. Murthy, Z. Phys. C42, 493 (1989); Erratum C44, 356 (1989);

H.Y. Cheng and S.N. Lai, Phys. Rev. D41, 91 (1990).

[12] A.P. Contogouris, B. Kamal, Z. Merebashvili and F.V. Tkachov, Phys. Lett. B304, 329 (1993); Phys. Rev. D48, 4092 (1993).

[13] L.E. Gordon and W. Vogelsang, Phys. Rev. D48, 3136 (1993).

[14] L.E. Gordon and W. Vogelsang, Univ. Dortmund report DO-TH 93/12, to appear in Phys. Rev. D49 (1994). 
[15] G. Altarelli and G.G. Ross, Phys. Lett. B212, 391 (1988);

G. Altarelli and W.J. Stirling, Particle World 1, 40 (1989);

A.V. Efremov and O.V. Teryaev, Dubna report E2-88-287 (1988), published in the proceedings of the Int. Hadron Symposium 1988, Bechyne, Czechoslovakia, 1988, eds. X. Fischer et al. (Czech. Academy of Science, Prague, 1989), p. 302.

[16] J. Alitti et al. (UA2 collab.), Phys. Lett. B263, 544 (1991).

[17] F. Abe et al. (CDF collab.), Phys. Rev. Lett. 68, 2734 (1992); Fermilab-PUB-92/001E, to appear in Phys. Rev. D; 'Direct Photon Results from CDF', contributed paper to the XIV. Int. Symposium on Lepton-Photon Interactions, Cornell University, Ithaca (NY), August 1993.

[18] In the following we shall usually drop the labels $A$ and $B$ which can stand either for a proton or an antiproton.

[19] RHIC Spin Collab., D. Hill et al., letter of intent RHIC-SPIN-LOI-1991;

G. Bunce et al., Particle World 3, 1 (1992);

G. Bunce, Conference Proceedings AIP, New York, Particles and Fields Series 42, 147 (1991).

[20] Note, however, that $E_{\gamma} d^{3} \sigma_{d i r}^{s u b} / d^{3} p_{\gamma}$ is free of initial state mass singularities.

[21] We shall soon see that the limit $\epsilon \rightarrow 0$ is not physical due to soft gluon emission.

[22] Needless to say that this also implies the use of next-to-leading order structure functions evolved in $\overline{\mathrm{MS}}$ in order to cancel the scheme dependence in the final result.

[23] A similar approximation was made for jet studies in refs. $[24,25,26]$.

[24] G. Sterman and S. Weinberg, Phys. Rev. Lett. 39, 1436 (1977).

[25] M. Furman, Nucl. Phys. B197, 413 (1982).

[26] F. Aversa, P. Chiappetta, M. Greco and J.Ph. Guillet, Phys. Rev. Lett. 65, 401 (1990).

[27] Our results for the subtraction cross sections $E_{\gamma} d^{3} \sigma_{d i r}^{s u b} / d^{3} p_{\gamma}$ and $E_{\gamma} d^{3} \sigma_{\text {frag }}^{\text {sub }} / d^{3} p_{\gamma}$ can be obtained in a FORTRAN code upon request via the e-mail address UPH405@unidozr.hrz.uni-dortmund.de.

[28] As was shown in [6], the dependence on $\delta$ is actually of the form $\sin ^{2}(\delta / 2)$. 
[29] F. Aversa, P. Chiappetta, M. Greco and J.Ph. Guillet, Phys. Lett. B210, 225 (1988); Phys. Lett. B211, 465 (1988); Nucl. Phys. B327, 105 (1989).

[30] Note that we keep all $\epsilon \ln \delta \ln \epsilon$ terms which should be far more important than $\epsilon \delta^{2} \ln \epsilon$.

[31] R.K. Ellis and J.C. Sexton, Nucl. Phys. B269, 445 (1986).

[32] G. 't Hooft and M. Veltman, Nucl. Phys. B44, 189 (1972);

P. Breitenlohner and D. Maison, Commun. math. Phys. 52, 11 (1977).

[33] Another shortcoming of present-day next-to-leading order calculations in spin physics is the lack of the two-loop anomalous dimensions for the polarized case, which are needed for a consistent evolution of the polarized parton distributions in next-toleading order.

[34] M. Glück, L.E. Gordon, E. Reya and W. Vogelsang, work in preparation.

[35] Note that only final state singularities are present in $E_{\gamma} d^{3} \sigma_{d i r}^{s u b} / d^{3} p_{\gamma}$ (see section 2). Therefore we do not have to introduce a soft cut-off $\delta_{s}$ which was necessary in the Monte Carlo calculation of [5] for the full isolated cross section $E_{\gamma} d^{3} \sigma_{d i r}^{i s o l} / d^{3} p_{\gamma}$.

[36] M. Glück, E. Reya and A. Vogt, Z. Phys. C53, 127 (1992).

[37] I. Abt et al. (H1-collab.), Nucl. Phys. B407, 515 (1993).

[38] M. Derrick et al. (ZEUS-collab.), Phys. Lett. B316, 412 (1993).

[39] This is so even though our Monte Carlo program only calculates $E_{\gamma} d^{3} \sigma_{d i r}^{\text {sub }} / d^{3} p_{\gamma}$, as opposed to the full Monte Carlo calculation of ref. [5] which relies on the numerical integration of even much larger regions of the phase space.

[40] G. Kramer and S.G. Salesch, DESY-report DESY 93-010 (1993), unpublished.

[41] M. Glück, E. Reya and A. Vogt, Phys. Rev. D48, 116 (1993). Of the two sets presented in this work we choose the one with an additional hadronic component at the input scale.

[42] R. Gastmans and T.T. Wu, 'The Ubiquitous Photon', Oxford Science Publications (1990). 


\section{Figure Captions}

Fig. 1: a: 'Direct' contribution to next-to-leading order prompt photon production with an additional parton in the isolation cone. b: Fragmentation contribution to nextto-leading order prompt photon production with an additional parton in the isolation cone.

Fig. 2: Definitions for the energies of the involved particles in a next-to-leading order fragmentation process with an additional parton in the isolation cone.

Fig. 3: Typical final state pole contribution in a next-to-leading order fragmentation process (cf. eq. (38)).

Fig. 4: a,b: Graphical representations for the two contributions to $d \hat{\sigma}_{\delta}^{g g \rightarrow g} / d v d w$ in the small-cone approximation, corresponding to eq. (41). The functions $\mathcal{P}_{g g}$ and $\mathcal{P}_{g q}$ were defined in eq. (40).

Fig. 5: a: The relative deviation $D$ defined in eq. (46) as a function of the isolation parameters $\delta$ and $\epsilon$ at $\sqrt{S}=1 \mathrm{TeV}, p_{T}=20 \mathrm{GeV}$ and $\eta=0$. We have chosen $\mu=M=p_{T}$, but $M_{f}=\delta p_{T}$. b: Same as in a, but for $p_{T}=50 \mathrm{GeV}$.

Fig. 6: The ratio $R_{d i r}$ of subtraction cross section and isolated cross section for the direct case, defined as in eq. (47), as a function of $p_{T}$. The solid line corresponds to using our approximation for $E_{\gamma} d^{3} \sigma_{d i r}^{s u b} / d^{3} p_{\gamma}$, whereas the dashed one refers to $E_{\gamma} d^{3} \sigma_{d i r}^{\text {sub }} / d^{3} p_{\gamma}$ calculated by the Monte Carlo program. The scales are chosen as in fig. 5 .

Fig. 7: The ratio $R_{\text {frag }}$ of subtraction cross section and isolated cross section for the next-to-leading order fragmentation case, defined as in eq. (48), as a function of $p_{T}$, calculated using our approximation method.

Fig. 8: The ratio $R$ of isolated fragmentation contribution and full isolated cross section as defined in eq. (49), as a function of $p_{T}$.

Fig. 9: Dependence of the full isolated cross section $E_{\gamma} d^{3} \sigma_{d i r}^{i s o l} / d^{3} p_{\gamma}+E_{\gamma} d^{3} \sigma_{f r a g}^{i s o l} / d^{3} p_{\gamma}$ on the energy resolution $\epsilon$ at fixed cone size $\delta=0.4$ for $p_{T}=20 \mathrm{GeV}, 50 \mathrm{GeV}$ and 100 GeV. As before we have chosen $\sqrt{S}=1 \mathrm{TeV}, \eta=0$ and the scales $\mu=M=p_{T}$, $M_{f}=\delta p_{T}$.

Fig. 10: Same as in fig. 9, but now as a function of the cone size $\delta$ at fixed $\epsilon=0.1$. 
Fig. 11: Dependence of the full isolated cross section $E_{\gamma} d^{3} \sigma_{d i r}^{i s o l} / d^{3} p_{\gamma}+E_{\gamma} d^{3} \sigma_{f r a g}^{i s o l} / d^{3} p_{\gamma}$ on the choice for the fragmentation scale $M_{f}$ for $p_{T}=20 \mathrm{GeV}$ and $50 \mathrm{GeV}$ at fixed $\delta=0.4$ and $\epsilon=0.1$. We have set $M_{f}=\xi \delta p_{T}$ and vary $\xi$ between 0.1 and 3 . As before we have chosen $\sqrt{S}=1 \mathrm{TeV}, \eta=0$, and the renormalization/(initial state) factorization scales $\mu=M=p_{T}$. 


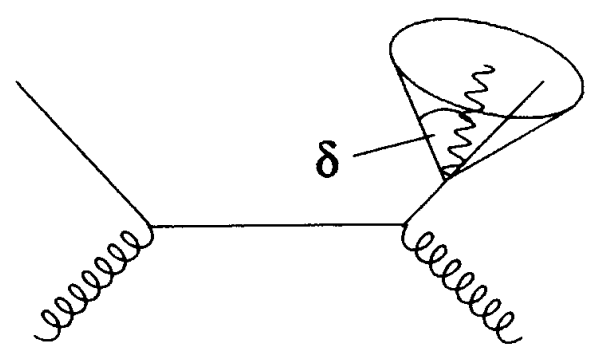

a

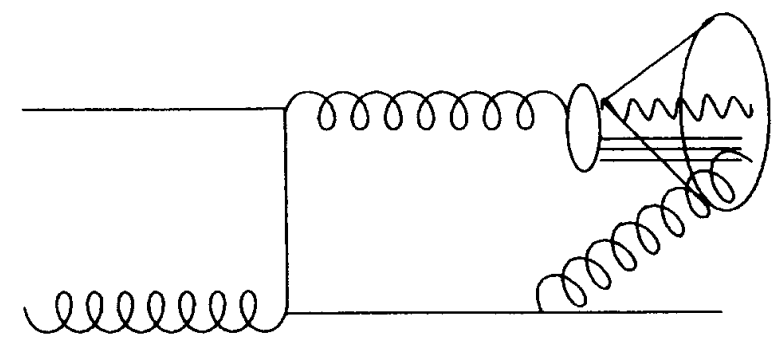

b

Fig. 1

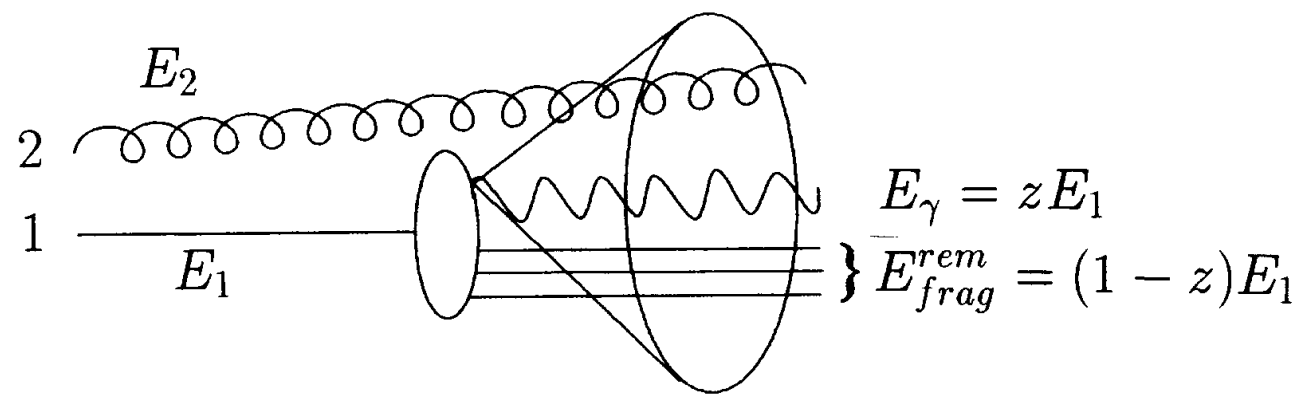

Fig. 2 


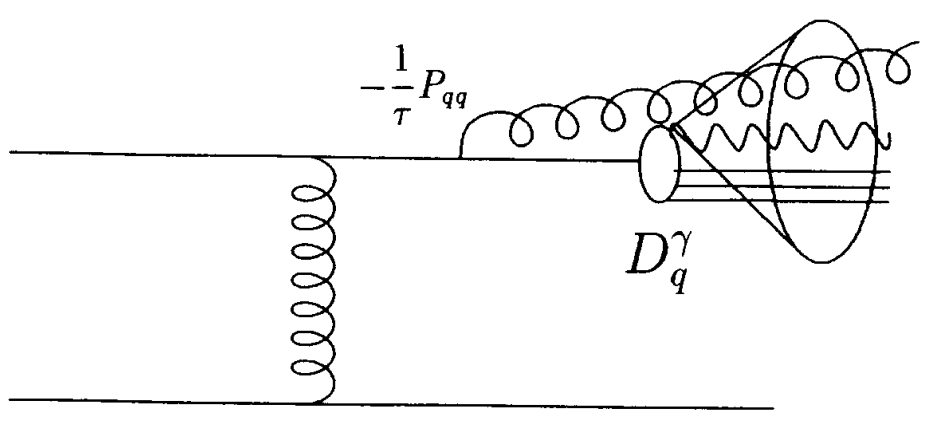

Fig. 3

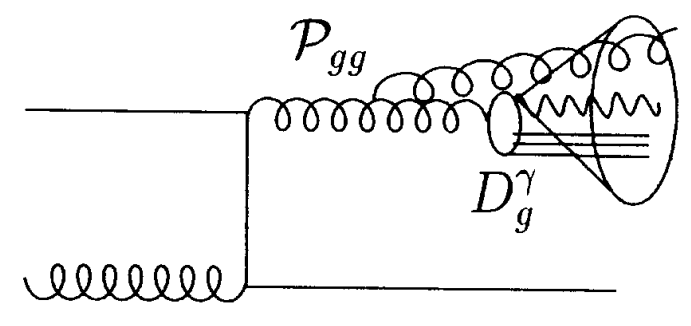

a

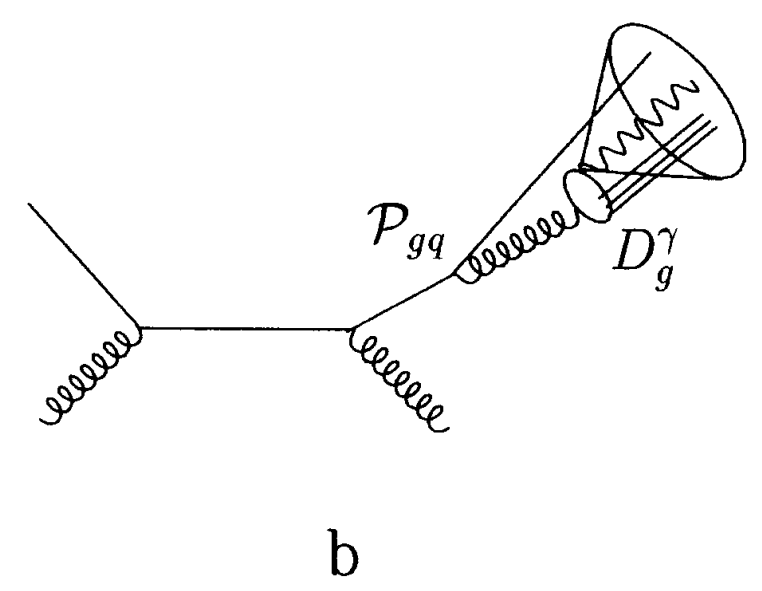

Fig. 4 


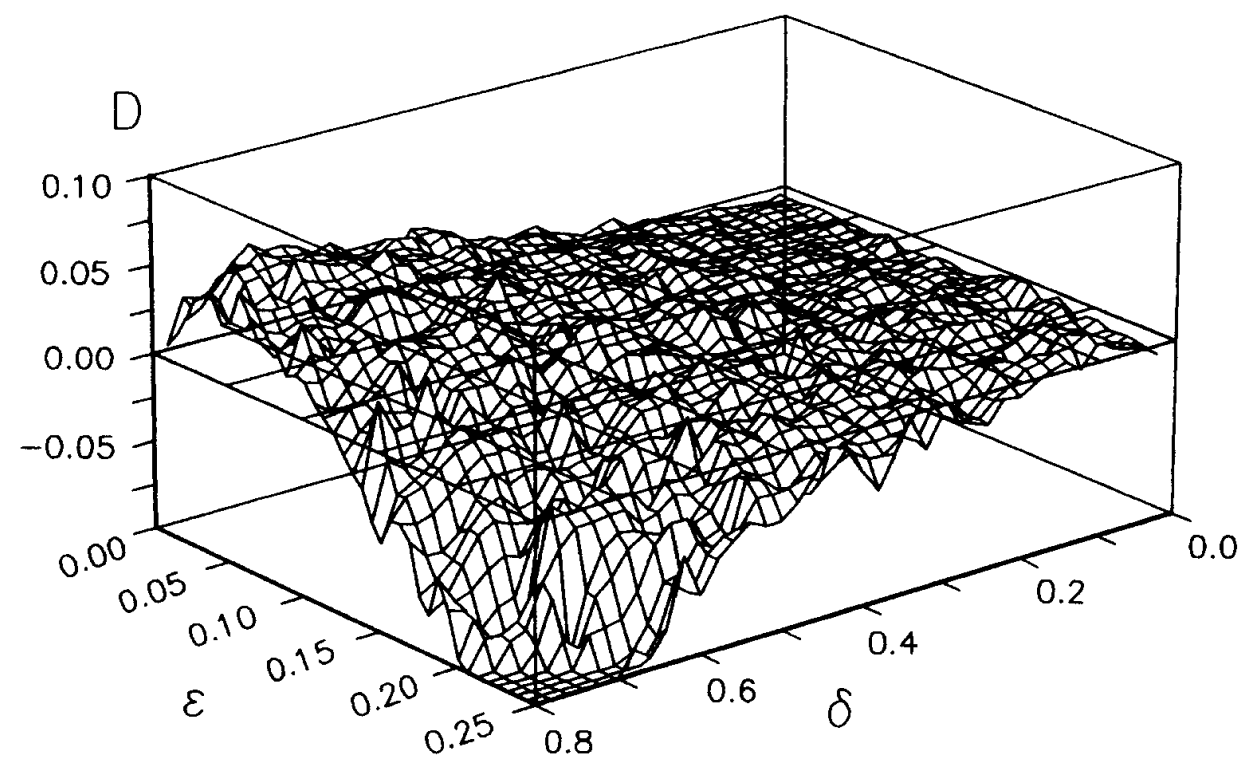

a

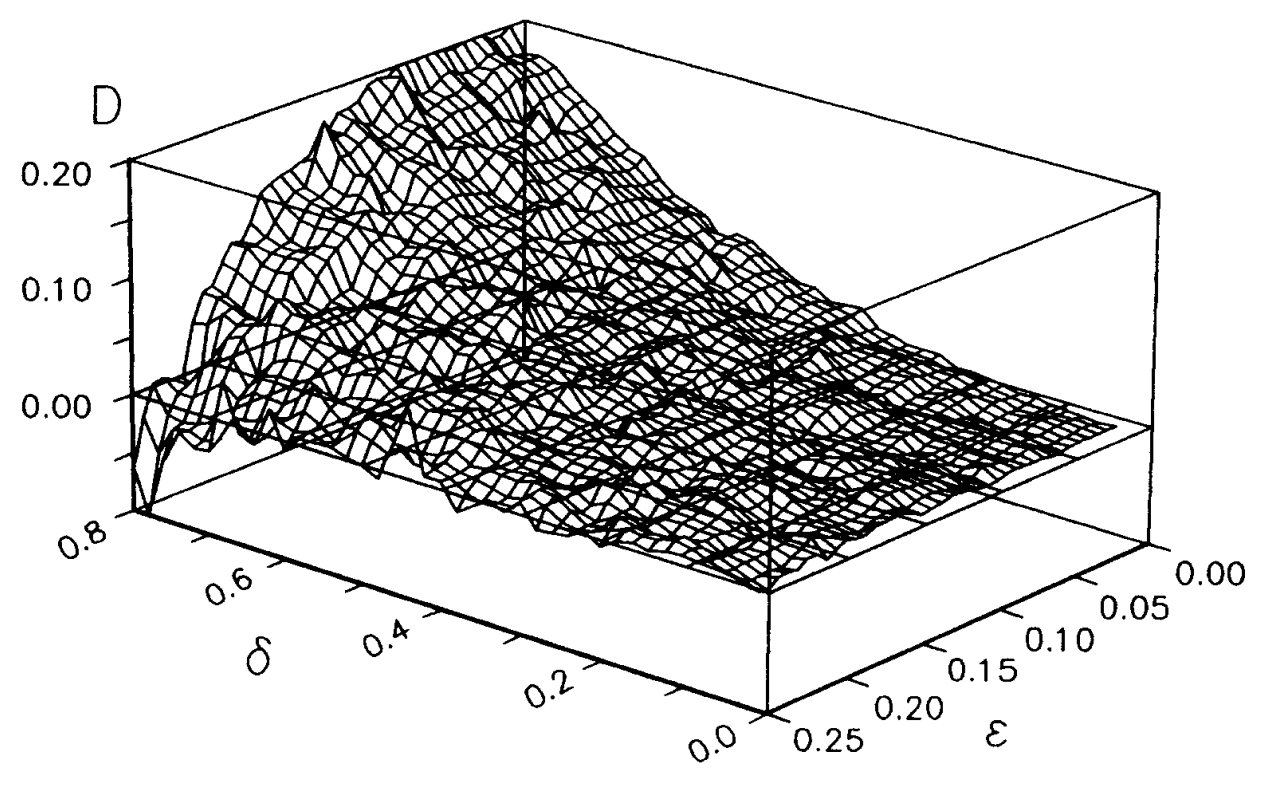

b

Fig. 5 


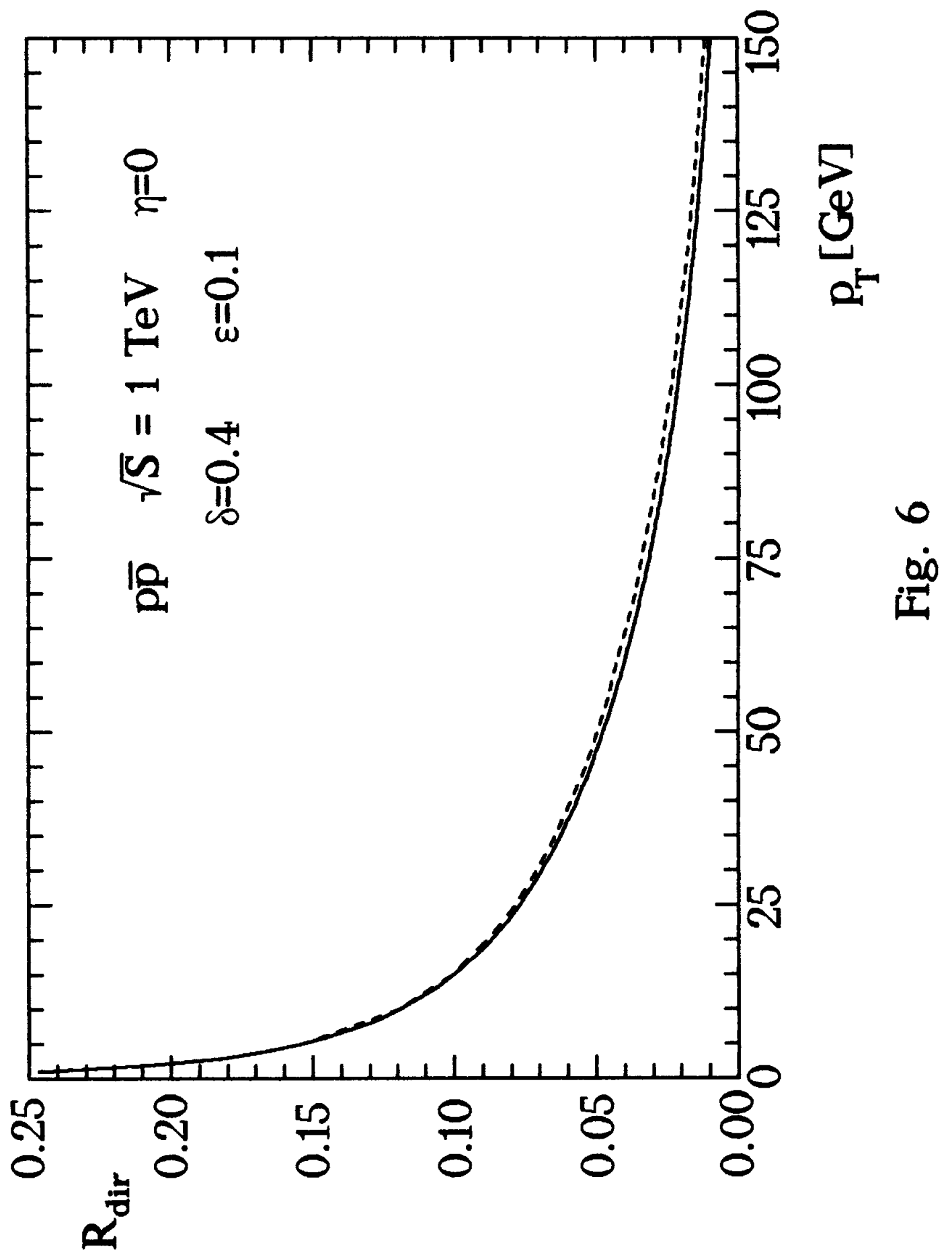




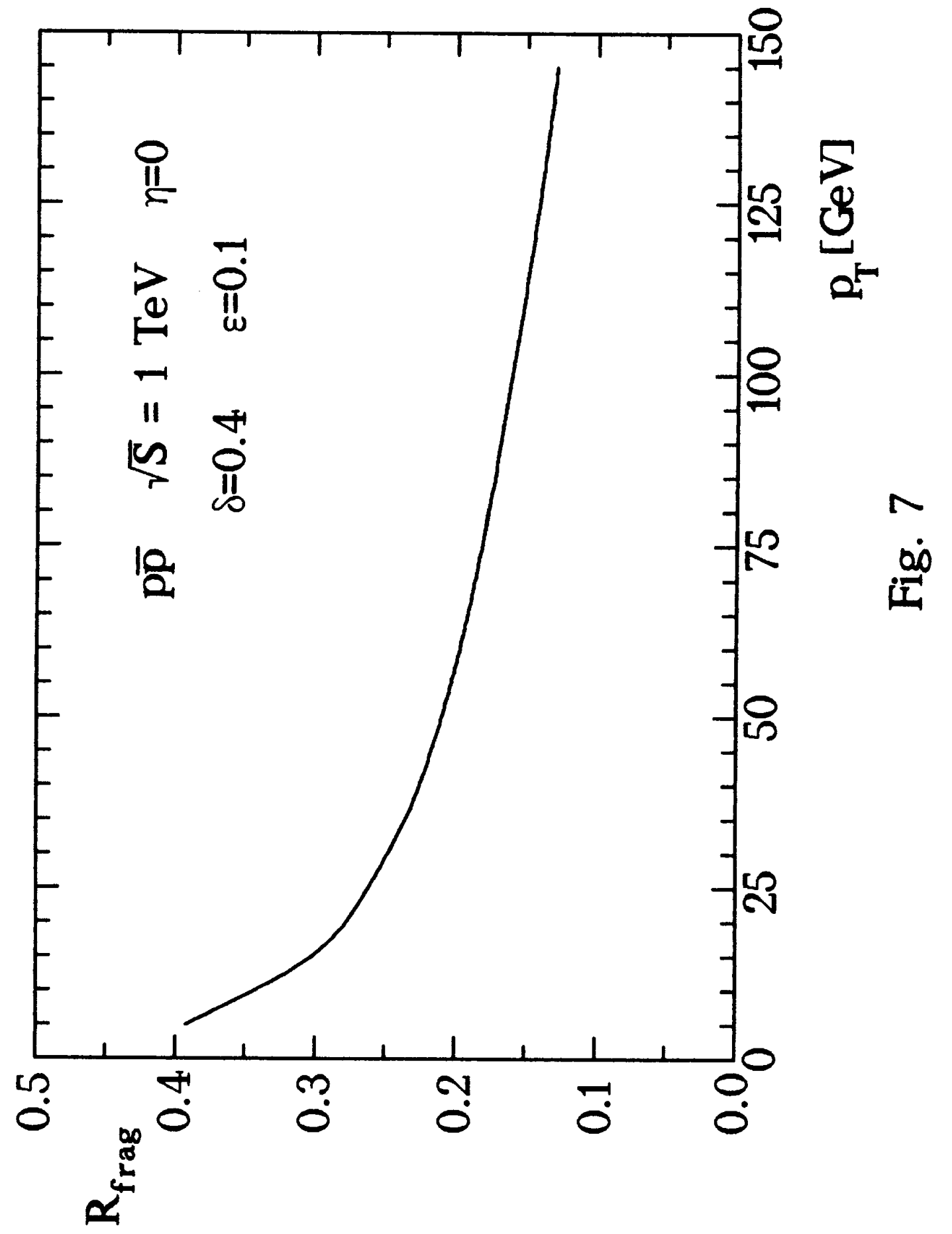




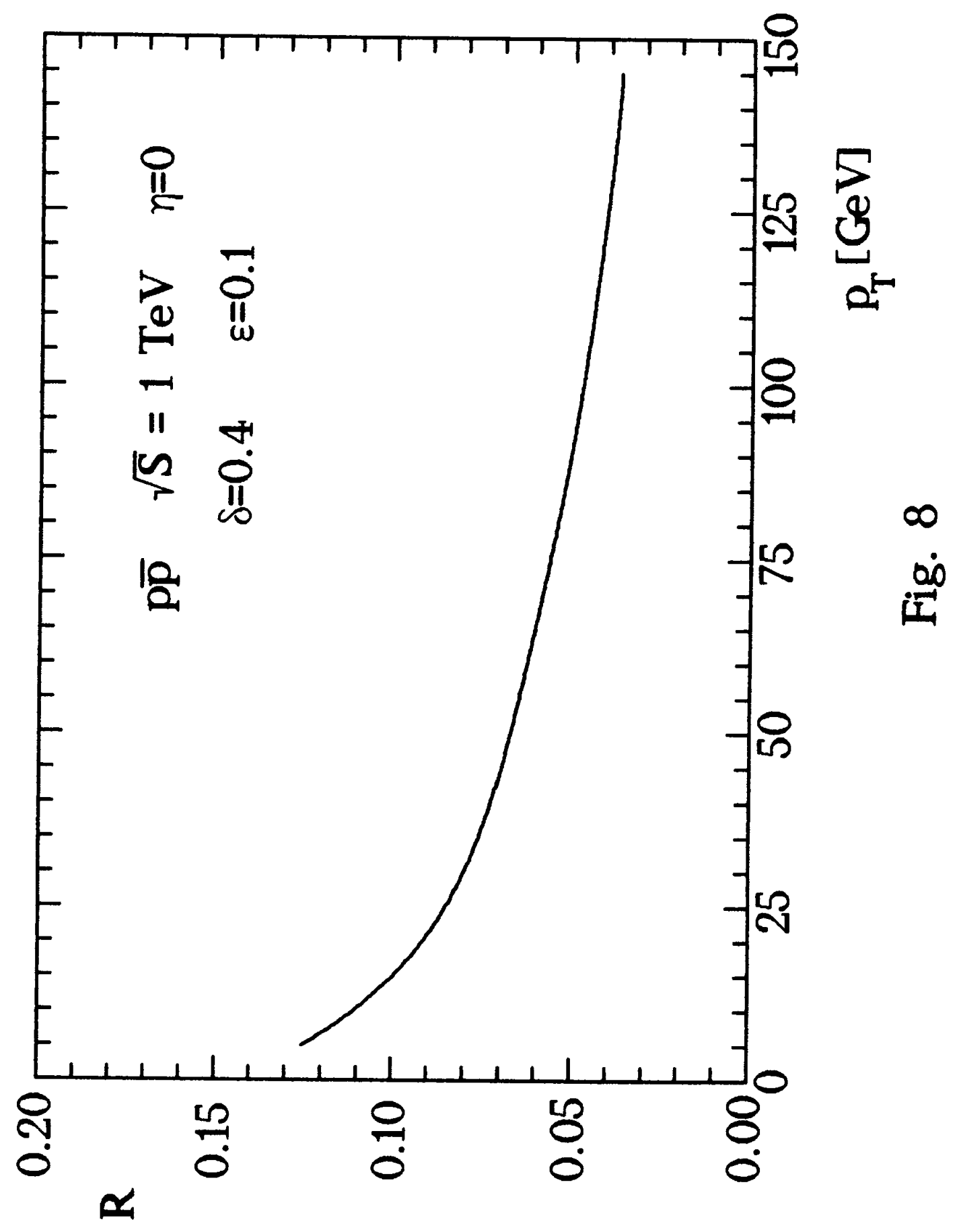




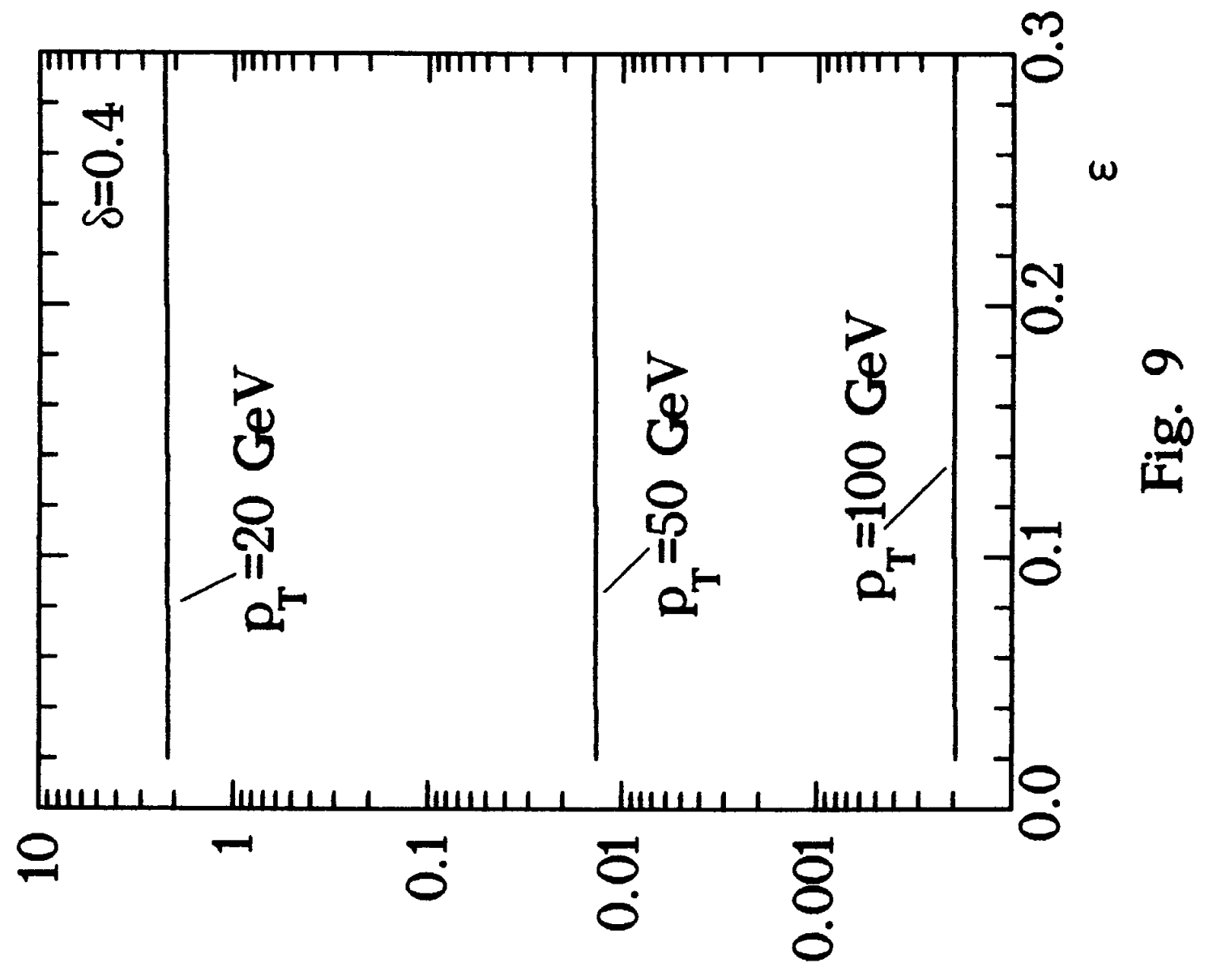

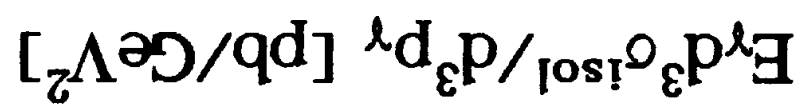




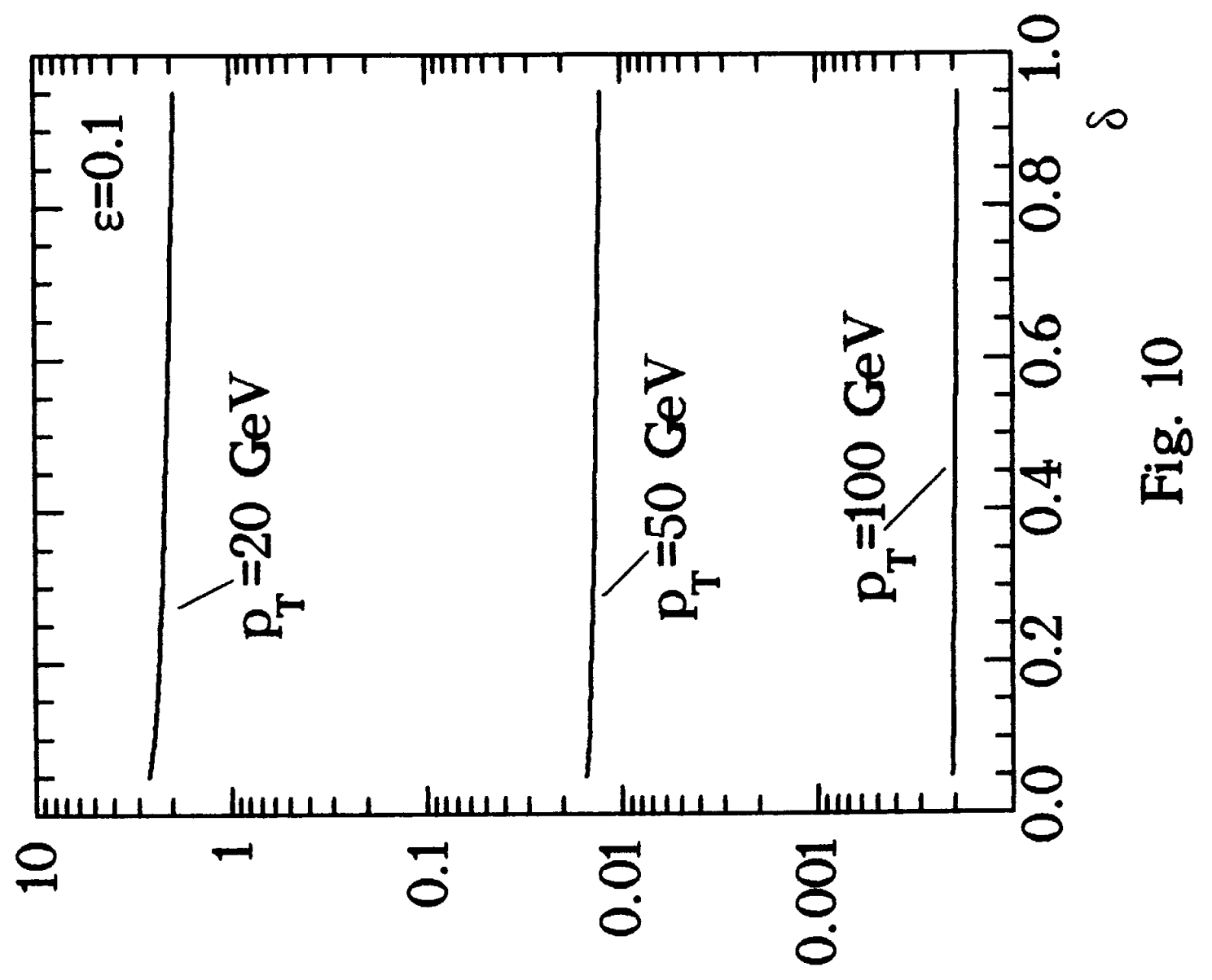

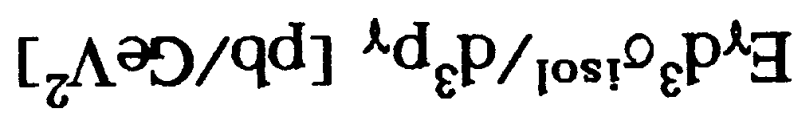




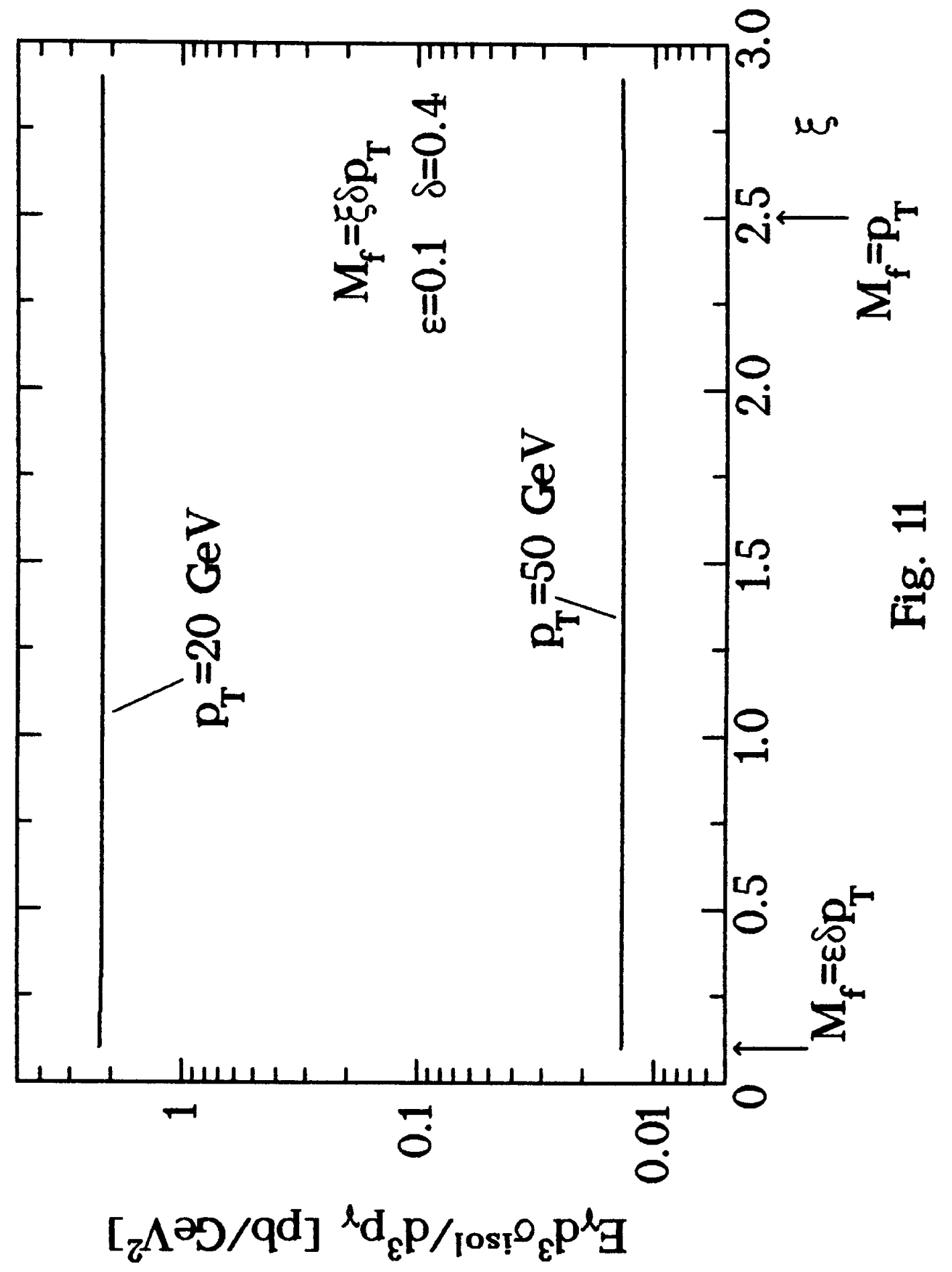

